\title{
A comparative in-silico analysis of autophagy proteins in ciliates
}

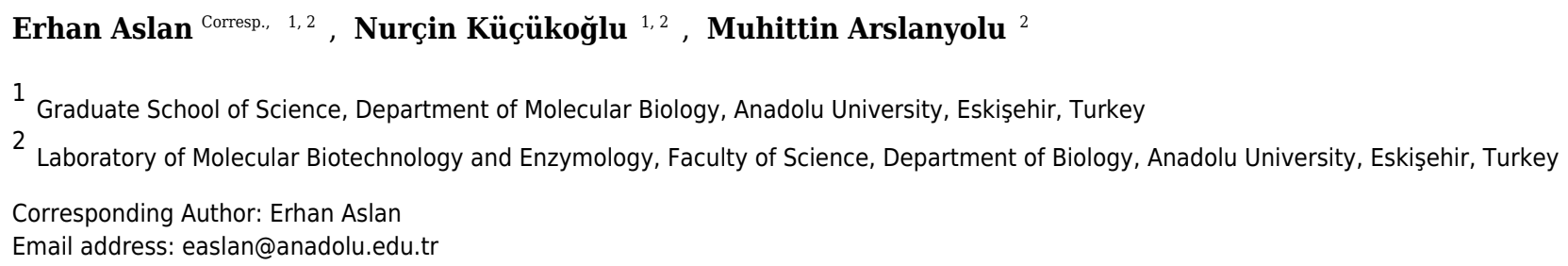

Autophagy serves as a turnover mechanism for the recycling of redundant and/or damaged macromolecules present in eukaryotic cells to re-use them under starvation conditions via a double-membrane structure, autophagosome. A set of eukaryotic genes called autophagy-related genes (ATGs) orchestrate this highly elaborative process. The existence of these genes and the role they play in different eukaryotes are wellcharacterized. However, little is known of their role in some eukaryotes, like ciliates. Here, we report the computational analyses of ATG genes in five ciliate genomes to understand their diversity. Our results show that Oxytricha trifallax is the sole ciliate which has a conserved Atg12 conjugation system (Atg5-Atg12-Atg16). Interestingly, Oxytricha Atg16 protein includes WD repeats in addition to its N-terminal Atg16 domain as is the case in multicellular organisms. Additionally, phylogenetic analyses revealed that E2-like conjugating protein Atg10 is only present in Tetrahymena thermophila. We fail to find critical autophagy components Atg5, Atg7 and Atg8 in the parasitic ciliate Ichthyophthirius multifiliis. Contrary to previous reports, we also find that ciliate genomes do not encode typical Atgl since all the candidate sequences lack an Atg1-specific C-terminal domain which is essential for Atgl complex formation. Consistent with the absence of Atg1, ciliates also lack other members of the Atg1 complex. However presence of Atg 6 in all ciliates examined here may rise the possibility that autophagosome formation could be operated through Atg6 in ciliates, since Atg6 has been shown as an alternative autophagy inducer. In conclusion, our results highlight that Atg proteins are partially conserved in ciliates. This may provide a better understanding for the autophagic destruction of the parental macronucleus, a developmental process also known as programmed nuclear death in ciliates. 


\section{A Comparative In-silico Analysis of Autophagy Proteins in 2 Ciliates}

3 Erhan Aslan ${ }^{1,2}$, Nurçin Küçükoğlu ${ }^{1,2}$, Muhittin Arslanyolu²

$4{ }^{1}$ Graduate School of Science, Department of Molecular Biology, Anadolu University, Eskişehir, Turkey

5 '2Laboratory of Molecular Biotechnology and Enzymology, Faculty of Science, Department of Biology,

6 Anadolu University, Eskişehir, Turkey

9 Corresponding Author:

10 Erhan Aslan

11 Anadolu University, Yunus Emre Campus, Faculty of Science, 26470, Eskişehir, Turkey

12 Email address: erhanaslan26@gmail.com 


\section{Abstract}

30 Autophagy serves as a turnover mechanism for the recycling of redundant and/or damaged

31 macromolecules present in eukaryotic cells to re-use them under starvation conditions via a

32 double-membrane structure, autophagosome. A set of eukaryotic genes called autophagy-related

33 genes $(\boldsymbol{A T G})$ orchestrate this highly elaborative process. The existence of these genes and the role

34 they play in different eukaryotes are well-characterized. However, little is known of their role in

35 some eukaryotes, like ciliates. Here, we report the computational analyses of $A T G$ genes in five

36 ciliate genomes to understand their diversity. Our results show that Oxytricha trifallax is the sole

37 ciliate which has a conserved Atg12 conjugation system (Atg5-Atg12-Atg16). Interestingly,

38 Oxytricha Atg16 protein includes WD repeats in addition to its N-terminal Atg16 domain as is the

39 case in multicellular organisms. Additionally, phylogenetic analyses revealed that E2-like

40 conjugating protein Atg10 is only present in Tetrahymena thermophila. We fail to find critical

41 autophagy components Atg5, Atg7 and Atg8 in the parasitic ciliate Ichthyophthirius multifiliis.

42 Contrary to previous reports, we also find that ciliate genomes do not encode typical Atg1 since

43 all the candidate sequences lack an Atg1-specific C-terminal domain which is essential for Atg1

44 complex formation. Consistent with the absence of Atg1, ciliates also lack other members of the

45 Atg1 complex. However presence of Atg6 in all ciliates examined here may rise the possibility

46 that autophagosome formation could be operated through Atg6 in ciliates, since Atg6 has been

47 shown as an alternative autophagy inducer. In conclusion, our results highlight that Atg proteins

48 are partially conserved in ciliates. This may provide a better understanding for the autophagic 
49 destruction of the parental macronucleus, a developmental process also known as programmed

50 nuclear death in ciliates.

51

52

53

54

55

56

57

\section{Introduction}

Autophagy is a catabolic lysosomal pathway in which long-lived proteins, cytoplasmic materials, organelles and even intracellular bacteria are eliminated from the cytoplasm in a selective or nonselective manner. Different types of autophagic processes have been defined. Among them macroautophagy (hereafter referred to as autophagy) requires a double-membrane vesicle called the autophagosome which encloses part of the cytoplasm to deliver material to the lysosomes (Xie \& Klionsky, 2007). More than thirty five autophagy-related genes (ATG) were identified by genetic screens in the yeast Saccharomyces cerevisiae and further analyses then showed that these genes are highly conserved among eukaryotes. Some of these genes, which are referred to as core autophagy machinery (Xie \& Klionsky, 2007), encode proteins that are responsible for autophagosome formation. These machinery members are categorized into three main groups; the Atg9 cycling system (Atg1 kinase complex, Atg2, Atg9, Atg13, Atg18, Atg27), the phosphatidylinositol 3-kinase (PI3K) complex (Atg6, Atg14, Vps15, Vps30, Vps38), and the ubiquitin-like protein conjugation system (Atg3, Atg4, Atg5, Atg7, Atg8, Atg10, Atg12, Atg16) (Xie \& Klionsky, 2007; Mizushima, Yoshimori \& Ohsumi, 2011; Jiang et al., 2012). Some of these proteins have particular significance due to their biological function. For instance, Atg1 complex activity is required for starvation-induced autophagy induction. Atg6, a member of the PI3K complex, has also been shown to be involved in autophagy induction in an Atg1-independent manner. In addition, its mammalian orthologue, Beclin-1 has been shown to be associated with 
72 many human diseases including different types of cancer and neurodegenerative diseases (Jiang \&

73 Mizushima, 2014). Atg8, on the other hand, is a marker protein responsible for both

74 autophagosome and puncta formation, and localization of the ATG8 gene product is used as a

75 diagnostic assay for autophagy (Mizushima \& Yoshimori, 2007). Though autophagic processes

76 are well-defined in myriad eukaryotic models, little is known about this pathway in ciliates.

77 Ciliates provide unique features for cellular and molecular biology related studies. These

78 eukaryotic microbes are best characterized by their binucleated genome architecture, known as

79 nuclear dimorphism (Orias, Cervantes \& Hamilton, 2011). There are two types of morphologically

80 and genetically different nuclei in the same cytoplasm. The polyploid macronucleus (MAC)

81 governs the cell phenotype by providing all necessary transcripts for biological functions and

82 serves as soma. The diploid micronucleus (MIC) is transcriptionally inert and functions as a

83 germline nucleus. Although there are some differences between species, ciliates have more or less

84 similar life cycles. For instance Ichthyophthirius multifiliis, a parasitic ciliate, requires a fish

85 species as a host for its life cycle (Coyne et al., 2011). Here we describe the life cycle of

86 Tetrahymena thermophila as a representative ciliate which contains three distinctive stages; (i)

87 growth, (ii) starvation and (iii) conjugation. (i) During vegetative growth cells proliferate by binary

88 fission. The MIC divides by mitosis whereas the MAC divides amitotically without spindle

89 formation. (ii) When cells are depleted of food, they enter the starvation stage. (iii) Conjugation is

90 the only sexual developmental stage of Tetrahymena, in which exchange of genetic material occurs

91 following meiosis. Conjugation is initiated by the physical interaction of two different mating-

92 types of starved cells. In a related ciliate, Paramecium tetraurelia, in addition to conjugation, there

93 is another way to reproduce sexually, called autogamy (self-fertilization), in which conjugative

94 events take place in a single cell without pair formation. In Tetrahymena, the MIC undergoes 
95 meiosis to produce four haploid nuclei. One of them selectively migrates to the anterior of the cell

96 and undergoes one round of mitosis. One of these mitotic products is exchanged between pairs,

97 while the remaining three meiotic products are degraded. After exchange, stationary and migratory

98 nuclei fuse to form a diploid zygotic nucleus, which corresponds to the fertilization of sperm and

99 eggs in metazoans. The zygotic nucleus then turns into four diploid products after two rounds of

100 mitotic division. Two of these nuclei migrate to the anterior of the cell to differentiate into new

101 MAC, whereas posteriorly positioned nuclei turn into MIC (Orias, Cervantes \& Hamilton, 2011).

102 Meanwhile, haploid meiotic products and poliploid parental MAC (old MAC) are degraded and

103 eliminated from the cytoplasm in an apoptotic and autophagic-fashion by a genetically and

104 developmentally programmed process, known as Programmed Nuclear Death (PND) (Akematsu,

105 Pearlman \& Endoh, 2010). Life cycle and nuclear events during conjugation in Tetrahymena are 106 illustrated in Fig. 1.

107 PND in ciliates is a highly elaborate process which includes apoptotic and autophagic-like 108 events. PND has been studied mostly with Tetrahymena (Akematsu, Pearlman \& Endoh, 2010;

109 Akematsu \& Endoh, 2010; Akematsu et al., 2014), and molecular mechanisms of PND in other

110 ciliates remain elusive. PND in Tetrahymena shares some phenotypic and biochemical

111 resemblance to apoptosis or programmed cell death (PCD) in higher eukaryotes. For instance,

112 chromatin condensation, loss of DNA integrity, genomic DNA degradation into high molecular

113 weight DNA and oligonucleosome-size DNA fragmentation (DNA laddering) are considered as

114 hallmarks of PCD, and all these are observed in PND of Tetrahymena. Consequently, several

115 apoptotic molecules and organelles have been linked with PND in Tetrahymena. Mitochondria,

116 for example, include a variety of apoptogenic factors (van Gurp et al., 2003). Kobayashi and Endoh

117 investigated the role of mitochondria in PND in Tetrahymena using two different dyes, and 
118 discovered that mitochondria and the degenerating MAC were co-localized in autophagosomes

119 (Kobayashi \& Endoh, 2005). Moreover, they detected a mitochondria-driven nuclease activity

120 functioning like mammalian EndoG during the DNA laddering process in Tetrahymena PND.

121 Recently, it has been shown that Tmn1, a ciliate specific mitochondrial nuclease, is responsible

122 for DNA laddering (Osada et al., 2014). Another apoptotic mitochondrial protein, AIF, is also

123 involved in Tetrahymena PND. Knocking out the AIF gene in MAC caused a four hour delay in

124 DNA fragmentation, but did not completely inhibit the PND (Akematsu \& Endoh, 2010). Recently,

125 we have detected increasing acidic DNase activity during late PND, implying a possible role for

126 lysosomal DNase II (Aslan \& Arslanyolu, 2015).

127 Recent studies in Tetrahymena also clearly revealed the involvement of Atg proteins in

128 PND. Liu and Yao, for instance, showed that two GFP-tagged Atg8 proteins preferentially

129 surround the degenerating parental MAC, but not newly-developing MAC in PND during 130 conjugation. In addition, these proteins have distinct roles during starvation, and $\triangle A T G 8$ cells

131 show clear delay in nuclear degradation (Liu \& Yao, 2012). Moreover, the role of class III

132 phosphatidylinositol 3-kinase (PI3K) in Tetrahymena has been recently studied (Akematsu et al.,

133 2014). It has been found that PI3K activity is required for autophagosome formation on the parental

134 MAC. In the knockdown mutant cells, the parental MAC escaped from the lysosomal pathway.

135 DNA fragmentation and final resorption of the parental nucleus are also found to be significantly

136 blocked with the loss of PI3K activity. Although there is no report describing whether any Atg

137 protein complexes exist in ciliates, the aforementioned studies show that Atg proteins have crucial 138 roles in PND.

139 On the other hand, use of autophagic dyes shows that PND also involves an unusual form 140 of autophagy which is different from yeast and mammalian autophagy. In yeast, autophagosome 
141 formation starts via accumulation of Atg proteins in a single dot-like site next to the vacuole, called

142 the phagophore assembly site (PAS), and the mammalian cells have the counterpart of PAS

143 (Shibutani \& Yoshimori, 2014). However, in Tetrahymena, after nuclear condensation, the

144 parental MAC changes its structure into an autophagosome membrane-like structure without

145 accumulation of a pre-autophagosomal structure (PAS-like) in the cytoplasm (Akematsu,

146 Pearlman \& Endoh, 2010).

147 In this study, our aim is to use a comparative analysis to expand current knowledge of Atg

148 proteins encoded in the genomes of model ciliates (Table 1). We find that, contrary to previous

149 reports (Rigden, Michels \& Ginger, 2009; Duszenko et al., 2011; Liu \& Yao, 2012), all ciliate

150 MAC genomes analyzed in this study lack a typical $A T G 1$ gene. We also discussed the presence

151 and absence of Atg proteins in ciliates in terms of PND.

152

153 Materials \& Methods

154 Sequence retrieval, BLAST and domain analyses

155 Five ciliate species, Tetrahymena thermophila, Paramecium tetraurelia, Ichthyophthirius

156 multifiliis, Oxytricha trifallax and Stylonychia lemnae are analyzed in this study (Table 1). All

157 these model ciliates have completed macronuclear genomes that are publicly available (Aury et

158 al., 2006; Eisen et al., 2006; Coyne et al., 2011; Swart et al., 2013; Aeschlimann et al., 2014). Atg

159 protein sequences were retrieved mainly from GenBank at http://www.ncbi.nlm.nih.gov/protein/

160 by probing S.cerevisiae Atg sequences against ciliates genomes in a PSI-BLAST analysis. When

161 no similar sequences were obtained, other Atg proteins from humans, P.pastoris and C.elegans

162 were also used as query. BLAST analyses were initially performed in ciliates, and then the

163 obtained candidate sequences were individually re-analyzed in species' genomes. An expected 
164 threshold (E-value) was set to 0.001 in all the BLAST-based analyses (Jiang et al., 2012; Földvári-

165 Nagy et al., 2014). Unless otherwise stated, Atg complexes that we mentioned throughout our

166 study come from yeast literature. The catalytic domains of candidate sequences were analyzed at

167 CDD (Marchler-Bauer et al., 2014), HMMER (Finn, Clements \& Eddy, 2011) and Pfam (Finn et

168 al., 2014). The presence or absence of Atg proteins in ciliates are presented by the Dot-blot BLAST

169 method (Field, Coulson \& Field, 2013). Accession numbers of Atg proteins found in this study are 170 given in Supplementary Table.

\section{Phylogenetic analysis}

172 Phylogenetic analyses were performed at online platform phylogeny.fr using the 'A la Carte' mode

173 (Dereeper et al., 2008). Multiple alignment was conducted with MUSCLE followed by alignment

174 curation with the 'remove positions with gaps' option. Unless otherwise stated maximum

175 likelihood algorithm was chosen to construct the phylogenetic tree with default settings. For 176 comparison, neighbor joining (NJ) and Bayesian trees were also calculated using default settings 177 at phylogeny.fr. The constructed ultimate trees are displayed and annotated by iTOL (Letunic \& 178 Bork, 2016) at http://itol.embl.de/.

179

180

mRNA expression analysis of $A T G$ genes of Tetrahymena and Paramecium

181 mRNA expression data of $A T G$ genes from Tetrahymena were obtained from the Tetrahymena

182 Functional Genomics Database (TetraFGD) (Miao et al., 2009) at http://tgd.ihb.ac.cn/. mRNA

183 expression data of $A T G$ genes from Paramecium were retrieved from the Paramecium Database

184 (Paramecium DB) (Arnaiz et al., 2010) at http://paramecium.cgm.cnrs-gif.fr/.

185 


\section{Results}

\section{Analysis of Atg9-Cycling system members and lack of Atg1 protein in ciliates}

188 The Atg9-Cycling system includes Atg9, Atg2, Atg18, Atg23, Atg27 and the Atg1 kinase complex 189 (Atg1, Atg13, Atg17, Atg29 and Atg31) in yeast. Atg9 is the sole transmembrane protein in the 190 core machinery and its activity is essential for autophagosome formation (Xie \& Klionsky, 2007).

191 It is known that Atg9 forms a complex with Atg2 and Atg18. As shown in Fig.2, all ciliates 192 analyzed in this study lack Atg9 and Atg2, but not Atg18 (Fig. 2a). We found two Atg18s in 193 oligohymenophorean ciliates Tetrahymena, Paramecium and Ichthyophthirius. Stichotrichous 194 ciliates Oxytricha and Stylonychia lemnae have four and five Atg18s encoded by their macronuclei, respectively (Fig. S1). reported a high number of $A T G 1$ genes in Tetrahymena and Paramecium, while yeast genomes have one and human encodes two (ULK1-2) (Rigden, Michels \& Ginger, 2009; Duszenko et al., 2011). Atg1 is a kinase domain containing protein and its activity is essential for autophagy induction and autophagosome formation in yeast and mammals. It contains three distinct domains; (i) a kinase domain at the $\mathrm{N}$ terminus, (ii) a proline-serine rich region at the center and (iii) a $\mathrm{C}$ terminal domain. Both in yeast and mammals, Atg1 complex members (Atg13, Atg17, Atg29, Atg31 in the yeast Atg1 complex; Atg13, Atg101 and FIP200 in the mammalian ULK1 complex) require the C-terminal domain of Atg1 to interact and form the complex (Chan \& Tooze, 2009) (Fig. 2b). Therefore, the presence of the C-terminal domain of Atg1 proteins appears crucial for function (Chan et al., 2009). Recently, Földvari-Nagy et al. analyzed non-unikont parasite genomes in terms of the presence or absence of Atg1 proteins (Földvári-Nagy et al., 2014). When they probed the complete yeast Atg1 sequence against 40 parasite species, they observed strong 
209 hits in return, but covering only the $\mathrm{N}$ terminal kinase domain. However, by using a truncated

210 version of the yeast Atg1 sequence which lacks the N-terminal kinase domain, no significant hits

211 were found. We applied the same experimental design in ciliate genomes and, when using yeast

212 Atg1 as query, we also observed a large number of significant hits in ciliates with a coverage of

213 only the N-terminal domain of the query (Fig. 2c). The truncated version of yeast and other Atg1

214 proteins, however, yielded no significant hits in ciliates (Fig. 2d). We conclude that these kinase

215 domain containing proteins should not be considered as Atg1 candidates without further

216 experimental analyses, since they all lack the important C-terminal domains. However, it is still

217 an open question whether any of these kinase domain containing proteins have role(s) in

218 autophagic processes in ciliates. Other components of this kinase complex (Atg1, Atg13, Atg17,

219 Atg29 and Atg31) are also absent in ciliates. Collectively, these results may imply that ciliates

220 might have an Atg1-independent pathway for autophagy induction and autophagosome formation.

\section{Analysis of PI3K complex members in ciliates}

223 Vacuolar protein sorting 34 (VPS34) gene encodes phosphatidylinositol 3-phosphate (PI3P) and is

224 required for autophagosome formation. In yeast, Vps34 forms two distinct complexes (Kihara et

225 al., 2001). Only complex I (Vps34, Atg14, Atg6/Vps30 and Vps15) is found to be involved in

226 autophagy (Fig. 3a). In complex II, Atg14 is replaced by Vps38, and this complex is involved in

227 endosome to-Golgi trafficking, not in autophagy. Except for Atg14, the remaining PI3K complex

228 members are found in ciliates. There is also experimental evidence for Vps $15-\mathrm{Vps} 34$ interaction

229 in Tetrahymena (Dr. Ronald E. Pearlman, pers. comm. 2016). In addition, Ichthyophthirius lacks

230 Vps15 (Fig. 3b, Fig. S2). The five ciliates discussed here have single Atg6 orthologue each. We

231 generated two phylogenetic trees of Atg6 with different algorithms. In the both maximum 
232 likelihood and bayesian trees, ciliate members found to be clustered together (Fig. 3c-d). The

233 presence of Atg6 in ciliates seems important since Atg6 has the ability to induce autophagy in an

234 Atg1 independent manner (Földvári-Nagy et al., 2014).

235 The mammalian counterpart of Atg6 is Beclin-1, and there are several non-yeast,

236 mammalian Beclin-1 interacting proteins (Kang et al., 2011). We researched ciliate genomes to

237 find the orthologues of these genes. We failed to find Ambra1, Dram, FIP200 or Atg101 in these

238 ciliates. However, surprisingly, UVRAG (UV radiation resistance-associated gene), a Beclin-1

239 binding protein which promotes autophagosome formation by activating Beclin-1 complex (Liang

240 et al., 2006; Afzal et al., 2015), is found only in Oxytricha among the ciliates analyzed. Mammalian

241 UVRAGs contain an Atg14 domain. However, we could not find any known domains in

242 Oxtricha's UVRAG in our domain analyses. We also found another non-yeast, Beclin-1

243 interacting autophagy protein, Vmp1 (Vacuole membrane protein 1), in all ciliates analyzed in this

244 study (Suppl. Table). Vmp1 is a transmembrane protein and is able to trigger autophagosome

245 formation in mammalian cells. It has been shown that Vmp1 interacts with Beclin-1 through its C-

246 terminal located Atg domain (Ropolo et al., 2007; Molejon et al., 2013). We found partial

247 conservation between Atg domain of human Vmp1 and ciliate members (Fig. S3). This kind of

248 interaction could compensate the absence of Atg1 in ciliates however, whether this is the case is

249 not currently known.

250

251 Analysis of ubiquitin-like conjugation system members in ciliates

252 In this group there are two conjugation systems; Atg8 and Atg12. The former includes Atg3, Atg4,

253 Atg7 and Atg8 interactions. All of these Atg proteins are found in all ciliates despite the

254 exceptional absence of $A T G 7$ and ATG8 in Ichthyophthirius (Fig. 4). The expression level of Atg8 
255 determines the size of the autophagosome (Feng et al., 2014). Atg8 is synthesized as a pro-enzyme

256 and an arginine residue at its C-terminus and is removed by Atg4, a cysteine protease, to expose a

257 glycine residue which is necessary for interaction with E1-like enzyme Atg7, for its activation

258 (Mizushima \& Yoshimori, 2007). Therefore, the C-terminal glycine residue is critical for

259 functional Atg8. We then analyzed Atg8 ciliate candidate sequences for the presence of

260 corresponding glycine residue (Fig. 4a). 3, 5, 6 and 22 genes met the criteria in Tetrahymena,

261 Stylonychia, Oxytricha and Paramecium, respectively. Interestingly, the Ichthyophthirius MAC

262 genome lacks $A T G 8$ and $A T G 7$. Atg7 is represented by one gene in the remaining ciliate species,

263 whereas other components of the Atg8 conjugation system differ greatly in numbers among species

264 (Fig S4). For example, Paramecium has more than 20 Atg8 and 10 Atg4 paralogues (Fig. 4b). This

265 situation is explained by the fact that Paramecium has undergone whole genome duplications

266 (WGD) during its evolutionary history (Duszenko et al., 2011). Other ciliates, whose evolutionary

267 pasts do not include WDG, have relatively fewer Atg4 and Atg8.

268 The Atg12 conjugation system, on the other hand, involves the interaction of Atg5, Atg7,

$269 \operatorname{Atg} 10, \operatorname{Atg} 12$ and Atg16. We found that $\operatorname{Atg} 12$ is present only in Oxytricha. Additionally,

270 Ichthyophthirius lacks Atg5 (Fig. 4c, Fig. S5). Our BLAST analysis revealed that several genes in

271 the MAC genomes of Oxytricha and Stylonychia have been annotated as Atg16 and Atg16-like

272 (Atg16L). In yeast, Atg16 forms a $350 \mathrm{kDa}$ complex with Atg12 and Atg5. In mice, however, this

273 complex is much larger, $800 \mathrm{kDa}$ (Fig. 4d). This difference is due to the long structure of

274 mammalian Atg16 members. While yeast Atg16 protein contains one Atg16 domain, mammalian

275 Atg16 proteins have seven WG repeat domains at their C-termini, in addition to the Atg16 domain

276 (Fig. 4e). For this reason, mammalian Atg16 proteins are referred to as Atg16-like (Atg16L)

277 (Mizushima et al., 2003). When we probed the ciliate genomes with yeast Atg16, no significant 
278 matches were found. BLAST analyses performed with mouse Atg16L resulted in a high number

279 of significant hits in the ciliate species. However, the majority of these hits showed sequence

280 similarity only to the WD40 domain of the query (Fig. 4e). On the other hand, some candidates

281 had significant similarity with the query covering both the Atg16 and WD40 domains. Domain

282 analyses for this Atg16 sequence showed that only the first hit (OXYTRI_20923) from Oxytricha

283 seems to be a true Atg16L, but not Atg16 judging from the presence of both Atg16 and multiple

284 WD40 domains (Fig. 4f, Fig. S6). Other significant hits, annotated as Atg 16 and Atg16-1 isoforms,

285 cannot be true Atg16, since they do not contain an Atg16 domain. Instead, they are simply WD40

286 repeat domains containing proteins (Fig. 4e). Based on these in silico observations, it seems

287 promising to study the Atg12 conjugation system in Oxytricha since as it is the sole ciliate species

288 carrying a conserved Atg12 conjugation system.

Previously Liu and Yao proposed TTHERM_00012980 from Tetrahymena as an Atg10

candidate (Liu \& Yao, 2012). However, our domain analysis showed that this gene likely encodes

an Atg3 protein (Fig. 5a). Cysteine residue in HPC motif in the catalytic domain and FLKC motif

in the C-terminal domain are found in this sequence, as well as in other Atg3 candidates (Fig. 5b).

293

During our BLAST analysis we could not find any Atg10-like proteins when Atg10 was used as a

probe. However in Atg3 BLAST analysis, we found three candidate sequences, two of them

possess Atg3-specific domain organization. The remaining candidate, on the other hand, contains

only single catalytic domain (TTHERM_01016200) (Fig. 5a). Atg3 and Atg10 have

"Autophagy_act_C" (PF03987) catalytic domain and catalytic sites of both proteins are similar

(Yamaguchi et al., 2012). We therefore decided to compare this sequence with yeast and human

300 (Fig. 5c). In order to clarify whether this candidate is an Atg3 or Atg10, we computed a 
301 phylogenetic tree using Atg3-Atg10 sequences (Fig. 5d, Fig. S7). Atg3 and Atg10s formed two

302 main clades as expected and TTHERM_01016200 was nested within the Atg10 clade. These

303 phylogenetic results, along with domain analysis, suggest that this protein is likely an Atg10

304 despite its low sequence similarity with experimentally shown Atg10s. No Atg10 found in other 305 ciliates.

306

307 Phylogeny of Atg4s and Atg8s in ciliates.

308 While the yeast genome encodes a single Atg4 and Atg8 proteins, there are multiple homologues

309 of these proteins in mammals like ciliates. It has been shown that among the four Atg4 proteins in

310 humans (Atg4a-d), Atg $4 \mathrm{~b}$ and $\operatorname{Atg} 4 \mathrm{c}$ are able to fully restore autophagic deficiencies in atg $4 \Delta$ yeast

311 cells, as revealed by complementation experiments (Marino et al., 2003). In addition, Atg4b has

312 the broadest capacity to use Atg8 proteins as substrate (Li et al., 2011). Mammalian Atg8

313 homologues are microtubule-associated protein 1 light chain 3 (LC3), $\gamma$-aminobutyric acid 314 receptor-associated protein (GABARAP) and Golgi-associated ATPase enhancer of $16 \mathrm{kDa}$

315 (GATE- 16) (Slobodkin \& Elazar, 2013). In order to understand the phylogenetic relationships of

316 Atg4s and Atg8s in ciliates with higher eukaryotes, maximum likelihood phylogenetic trees were

317 constructed (Fig. 6). In the Atg4 phylogeny (Fig. 6a), two main clades were observed. The first

318 clade (collapsed nodes, triangle) consists of only ciliate-specific Atg4 members. However, the

319 other clade includes yeast Atg4, mammalian Atg4s and several Atg4 proteins from stichotrichous

320 ciliates (except TTHERM_00622890, which belongs to Tetrahymena). We also constructed a

321 phylogenetic tree for Atg8s (Fig. 6b). Since Atg8 and Atg12 are phylogenetically related, the tree

322 was rooted with a group of Atg12 proteins (Williams et al., 2009). Consistent with our BLAST

323 and domain analyses, Oxytricha Atg12 (OXYTRI_18637) nested within the Atg12 clade, which 
324 further confirms that it is a real Atg12 orthologue. In addition to outgroup, the topology of the tree

325 consists of four other main clades. While three of these clades include only members from

326 Paramecium and Paramecium+Tetrahymena, the last remaining clade includes Atg8 members

327 from Oxytricha, Stylonychia, yeast and humans. These results indicate that the Atg4s and Atg8s

328 of stichotrichous ciliates are phylogenetically related to the Atg4s and Atg8s of higher eukaryotes.

330 Complete absence of non-core Atg proteins in ciliates.

331 In addition to core machinery members, several gene products are only required for non-selective

332 and pathway-specific (selective) autophagy, while core proteins play a role in both selective and

333 non-selective autophagy. For instance, an Atg17-Atg19-Atg31 complex is specifically required for

334 non-selective autophagy in Saccharomyces cerevisiae (Kabeya et al., 2009). On the other hand, a

335 set of proteins (Atg11, Atg19-21, Atg23-28, Atg30, Atg32 and Atg33) are required for selective

336 autophagy, such as the Cvt pathway, pexophagy and mitophagy (Lynch-Day \& Klionsky, 2010).

337 We failed to identify any of these genes in ciliates, which indicates that ciliates only possess a part 338 of the core $A T G$ genes.

mRNA expression analysis of $\boldsymbol{A T G}$ genes from Tetrahymena and Paramecium.

341 In order to get an idea about the role of autophagy genes in the degradation of the parental MAC,

342 we analyzed mRNA expression profiles of $A T G$ genes during Tetrahymena conjugation and

343 Paramecium autogamy. These are the only two ciliates in this study that have publicly available

344 microarray data; therefore our comparative analysis is limited to these two ciliates (Miao et al.,

345 2009; Arnaiz et al., 2010). We found that ATG3s and ATG10 in Tetrahymena reach their maximum

346 expression levels in conjugation with different timing. For instance, TTHERM_01050620 is 
347 expressed constitutively at a quite high level with a peak of expression at the initiation of

348 starvation, reaching its peak expression at the fourth hour of conjugation, when meiosis II is

349 completed. The expression level of TTHERM_00012980 is quite constant throughout the life

350 cycle, but slightly higher at eight hours of conjugation in which PND also starts. The only Atg10-

351 like protein in ciliates, TTHERM_01016200, is expressed at quite a low level and shows its

352 maximum expression at six hours of conjugation, which coincides with nuclear exchange. (Fig.

353 7a). Collectively, these results show that expression of ATG3s and ATG10 in Tetrahymena are

354 differentially regulated during the life cycle, depending on the nuclear stages. Since expression

355 levels of these genes are higher between four to eight hours of conjugation, it is possible to

356 postulate that TTHERM_01050620 (Atg3) and TTHERM_01016200 (Atg10) may be involved in

357 the degradation of meiotic products and TTHERM_00012980 (Atg3) may take some roles in the

358 parental MAC turnover during Tetrahymena conjugation. On the other hand, expression levels of

359 ATG3s in Paramecium autogamy show no significant change throughout the life cycle.

360 Among the five ATG4 genes, only TTHERM_00526270 shows conjugation-specific

361 expression at eight hours when PND starts (Fig. 7a). Interestingly, two of ten $A T G$ genes of

362 Paramecium (GSPATG00027262001 and GSPATT00037390001) also show significant

363 expression during autogamy at meiosis and parental MAC fragmentation stages (Fig. 7b).

364 Therefore, we suggest that Atg4 may function during PND of ciliates.

365 Liu and Yao showed that ATG8s in Tetrahymena have conjugation-specific expression

366 profiles covering the four to eight hours of conjugation in which the three meiotic products and

367 parental MAC are eliminated (Liu \& Yao, 2012). Among the twenty two ATG8 genes of

368 Paramecium, we found that only GSPATT00020835001 has significant expression during 
369 autogamy, not only at the meiosis and fragmentation stages, but also at the remaining stages of

370 autogamy, such as development 1, 2 and 3 (Fig. 7b).

372 Discussion

373 In this study, we bioinformatically analyzed Atg proteins in ciliates. Our study is a continuing

374 effort that deals with the evolutionary differentiation of Atg proteins in ciliates, since several

375 groups have previously analyzed Atg proteins in protists. Ridgen et al. reported the first thorough

376 bioinformatics analysis in protists, including Tetrahymena and Paramecium, with evolutionary

377 aspects of the presence and absence of $A T G$ genes (Rigden, Michels \& Ginger, 2009). Duszenko

378 et al. also reviewed autophagy in protists in a similar approach to Ridgen et al (Duszenko et al.,

379 2011). Brennand et al. advanced autophagy knowledge in parasitic protists, mainly for possible

380 drug targeting as well as for the evaluation of the distribution of $A T G$ genes (Brennand et al.,

381 2011). However, our current study possesses certain novelties, which differentiates this paper from

382 previous work as discussed below.

383 In the aforementioned studies, authors have focused mainly on parasitic protists with least

384 emphasis on free-living protists, such as Tetrahymena and Paramecium. Later on, additional

385 macronuclear genomes of other model ciliates, Oxytricha (Swart et al., 2013), Ichthyophthirius

386 (Coyne et al., 2011) and Stylonychia (Aeschlimann et al., 2014), were fully sequenced. Thus, it

387 was essential to analyze Atg proteins in the light of newly-sequenced ciliate MAC genomes.

388 Remarkably, previous studies reported the presence of multiple ATG1 genes in

389 Tetrahymena and Paramecium. For example, Liu and Yao determined sixty candidate ATG1 genes

390 in Tetrahymena (Liu \& Yao, 2012). However, as with others, they also used the complete Atg1

391 sequence in their BLAST analysis as a query. Atg1 is a kinase domain containing protein and, 
392 considering the fact that these kinds of proteins are common in ciliates (Eisen et al., 2006), it is

393 therefore likely that these $A T G 1$ candidate genes, detected by Liu and Yao, may be false positive,

394 since all these sequences lack a critical C-terminal domain, which is essential for the formation of

395 the Atg1 complex (Chan \& Tooze, 2009; Földvári-Nagy et al., 2014). However, we cannot exclude

396 the possibility that these enzymes might have roles during PND and/or any autophagic-like

397 processes in ciliates.

398 In agreement with previous studies, our results also show that several key Atg proteins,

399 such as Atg1, Atg9 and Atg14, are absent, not only in Tetrahymena and Paramecium, but also in

400 ciliates. This could be at least one of the predictable reasons why autophagosome formation in

401 PND of Tetrahymena differs from mammalian and yeast autophagy. Akematsu et al. showed the

402 occurrence of autophagosome-like structures only on parental MAC, not on newly-developing

403 MAC, without accumulation of PAS-like membrane structures. Their transmission electron

404 microscopy analysis revealed that parental MAC does not possess a double-layered membrane

405 derived from PAS, which is the case in typical macroautophagy (Akematsu, Pearlman \& Endoh, 406 2010). In yeast, the Atg1 kinase complex is located at the PAS. Similarly, PAS is one of the 407 localization sites of Atg9 in yeast (Feng et al., 2014). Atg14, on the other hand, is responsible for 408 directing the PtdIns3K complex to the PAS (Obara, Sekito \& Ohsumi, 2006). However, genes 409 coding these proteins seem to be absent in ciliate MAC genomes. Considering the critical roles of 410 these proteins in autophagosome formation, it would be reasonable to assume that a lack of 411 canonical autophagosome formation during PND in Tetrahymena may be due to the absence of 412 critical core machinery components, such as Atg1, Atg9 and Atg14. However, it is worth noting 413 that some autophagosome-like structures have been observed in starved Tetrahymena (Liu \& Yao, 
414 2012) and T.pyriformis (Nilsson, 1984). However, autophagosome formation during starvation

415 and/or conjugation in other ciliates still remains elusive.

416 Previously, it has been shown that genetic defects in ATG8 and VPS34 genes causes

417 abnormal nuclear positioning during PND in Tetrahymena (Liu \& Yao, 2012; Akematsu et al.,

418 2014). In addition, Atg8-2 has been shown to be responsible for the selective marking of parental

419 MAC for degradation in Tetrahymena. Interestingly, our analysis revealed that ATG8 (also ATG5,

$420 A T G 7$ and VPS15 which are present in other ciliates) is not present in the Ichthyophthirius genome.

421 Given that Atg8 is required for autophagosome formation and is used to monitor autophagy, the

422 absence of Atg8 in Ichthyophthirius can raise the possibility that autophagy, in this parasitic ciliate,

423 may not be operated in a canonical manner. Indeed, it is highly questionable whether

424 Ichthyophthirius has sexual reproduction since conjugation in Ichthyophthirius has not been

425 studied in depth to date. Although in a very recent study, population genetics data showed that this

426 organism also reproduces sexually, but that conjugation-related genes in this ciliate are poorly-

427 conserved compared to Tetrahymena, suggesting that mechanical differences between

428 Ichthyophthirius and other ciliates may exist (MacColl et al., 2015). Therefore, currently it is

429 difficult to speculate about the role of $A T G$ genes during PND in Ichthyophthirius. However,

430 further analyses on $A T G$ genes in this parasitic ciliate may be helpful for possible drug targeting

431 to combat with the white spot disease, caused by Ichthyophthirius in fresh water fish.

432 Comparative mRNA expression profile analysis showed that the expression of several ATG

433 genes is up-regulated during Tetrahymena conjugation and Paramecium autogamy (Fig. 7).

434 Experimental and statistical approaches are important in microarray studies, especially when

435 deciphering microarray data that comes from two different organisms. For example, in a

436 Paramecium microarray study, the authors performed TREAT analysis, a bioinformatics 
437 approach, to test significance relative to a fold-change threshold in microarray experiments

438 (Mccarthy \& Smyth, 2009; Arnaiz et al., 2010). According to this approach, we found only three

439 genes with significant expression change in Paramecium autogamy (2 ATG4 and single ATG8)

440 (Fig. 7b). However, such a bioinformatics analysis has not been performed in a Tetrahymena

441 microarray study. For this reason, we subjectively determined the differentially expressed $A T G$

442 genes during the conjugation of Tetrahymena. Objectively, it is still possible that statistically or

443 subjectively significant expression changes may not be biologically meaningful. Likewise, a

444 statistically insignificant expression profile may be biologically meaningful. To clarify this, further

445 experimental evaluations are required.

446

447 Conclusions

448 In this study, we bioinformatically analyzed Atg proteins in ciliates. Ciliates constitute as model 449 organisms for plenty of molecular and cellular studies, including telomere biology, self-splicing 450 introns and RNAi-guided genome shaping. Recent studies have shown that different types of apoptotic and autophagic mechanisms exist during the destruction of the parental nucleus in Tetrahymena. Starvation is not only a strong physiological inducer of autophagy, but also a physiological and developmental stage of ciliates. This provides a good opportunity to study crosstalk between apoptosis and autophagy. The presence of certain genes solely in Oxytricha (like ATG12, ATG16L and UVRAG) and the absence of certain critical genes in Ichthyophthirius (like ATG5, ATG7 and ATG8) might open new platforms in the understanding of the role of autophagy in a broader sense. As a conclusion, the results presented in this study could be used to understand the novel functions of autophagy in different taxa. 
460 Acknowledgement

461 We owe special thanks to Dr. Ronald E. Pearlman (York University, Toronto, Canada) for his

462 critical reading of the manuscript. During peer-review period of our manuscript, The Nobel Prize

463 in Physiology or Medicine 2016 was awarded to Dr. Yoshinori Ohsumi "for his discoveries of

464 mechanisms for autophagy". On this opportunity, we would like to dedicate our paper to Dr.

465 Ohsumi for introducing us with autophagy.

466

467 References

468

469

470

471

472

473

474

475

476

477

478

479

480

481

482

483

484

485

486

487

488

489

490

491

492

493
Aeschlimann SH., Jönsson F., Postberg J., Stover N a., Petera RL., Lipps H-J., Nowacki M., Swart EC. 2014. The Draft Assembly of the Radically Organized Stylonychia lemnae Macronuclear Genome. Genome biology and Evolution 6:1707-23. DOI: $10.1093 / \mathrm{gbe} / \mathrm{evu} 139$.

Afzal S., Hao Z., Itsumi M., Abouelkheer Y., Brenner D., Gao Y., Wakeham A., Hong C., Li WY., Sylvester J., Gilani SO., Brüstle A., Haight J., You-Ten AJ., Lin GHY., Inoue S., Mak TW. 2015. Autophagy-independent functions of UVRAG are essential for peripheral naive T-cell homeostasis. Proceedings of the National Academy of Sciences 112:1119-1124. DOI: 10.1073/pnas.1423588112.

Akematsu T., Fukuda Y., Attiq R., Pearlman RE. 2014. Role of class III phosphatidylinositol 3kinase during programmed nuclear death of Tetrahymena thermophila. Autophagy 10:209225. DOI: $10.4161 /$ auto.26929.

Akematsu T., Endoh H. 2010. Role of apoptosis-inducing factor (AIF) in programmed nuclear death during conjugation in Tetrahymena thermophila. BMC cell biology 11:13. DOI: 10.1186/1471-2121-11-13.

Akematsu T., Pearlman RE., Endoh H. 2010. Gigantic macroautophagy in programmed nuclear death of Tetrahymena thermophila. Autophagy 6:901-911. DOI: 10.4161/auto.6.7.13287.

Arnaiz O., Goût J-F., Bétermier M., Bouhouche K., Cohen J., Duret L., Kapusta A., Meyer E., Sperling L. 2010. Gene expression in a paleopolyploid: a transcriptome resource for the ciliate Paramecium tetraurelia. BMC Genomics 11:547. DOI: 10.1186/1471-2164-11-547.

Aslan E., Arslanyolu M. 2015. Identification of neutral and acidic deoxyribonuclease activities in Tetrahymena thermophila life stages. European Journal of Protistology 51:173-185. DOI: 10.1016/j.ejop.2015.02.004.

Aury J-M., Jaillon O., Duret L., Noel B., Jubin C., Porcel BM., Ségurens B., Daubin V., Anthouard V., Aiach N., Arnaiz O., Billaut A., Beisson J., Blanc I., Bouhouche K., Câmara F., Duharcourt S., Guigo R., Gogendeau D., Katinka M., Keller A-M., Kissmehl R., Klotz 
494

495

496

497

498

499

500

501

502

503

504

505

506

507

508

509

510

511

512

513

514

515

516

517

518

519

520

521

522

523

524

525

526

527

528

529

530

531

532

533

534

535

C., Koll F., Le Mouël A., Lepère G., Malinsky S., Nowacki M., Nowak JK., Plattner H., Poulain J., Ruiz F., Serrano V., Zagulski M., Dessen P., Bétermier M., Weissenbach J., Scarpelli C., Schächter V., Sperling L., Meyer E., Cohen J., Wincker P. 2006. Global trends of whole-genome duplications revealed by the ciliate Paramecium tetraurelia. Nature 444:171-178. DOI: 10.1038/nature05230.

Brennand A., Gualdrón-López M., Coppens I., Rigden DJ., Ginger ML., Michels P a M. 2011. Autophagy in parasitic protists: Unique features and drug targets. Molecular and Biochemical Parasitology 177:83-99. DOI: 10.1016/j.molbiopara.2011.02.003.

Chan EYW., Longatti A., McKnight NC., Tooze SA. 2009. Kinase-inactivated ULK proteins inhibit autophagy via their conserved C-terminal domains using an Atg13-independent mechanism. Molecular and cellular biology 29:157-171. DOI: 10.1128/MCB.01082-08.

Chan EY., Tooze S a. 2009. Evolution of Atg1 function and regulation. Autophagy 5:758-765. DOI: 8709 [pii].

Coyne RS., Hannick L., Shanmugam D., Hostetler JB., Brami D., Joardar VS., Johnson J., Radune D., Singh I., Badger JH., Kumar U., Saier M., Wang Y., Cai H., Gu J., Mather MW., Vaidya AB., Wilkes DE., Rajagopalan V., Asai DJ., Pearson CG., Findly RC., Dickerson HW., Wu M., Martens C., Van de Peer Y., Roos DS., Cassidy-Hanley DM., Clark TG. 2011. Comparative genomics of the pathogenic ciliate Ichthyophthirius multifiliis, its free-living relatives and a host species provide insights into adoption of a parasitic lifestyle and prospects for disease control. Genome Biology 12:R100. DOI: 10.1186/gb-2011-12-10-r100.

Dereeper A., Guignon V., Blanc G., Audic S., Buffet S., Chevenet F., Dufayard JF., Guindon S., Lefort V., Lescot M., Claverie JM., Gascuel O. 2008. Phylogeny.fr: robust phylogenetic analysis for the non-specialist. Nucleic acids research 36:465-469. DOI: 10.1093/nar/gkn180.

Duszenko M., Ginger ML., Brennand A., Gualdrón-lópez M., Colombo M., Coombs GH., Coppens I., Jayabalasingham B., Langsley G., Castro SL De., Menna-barreto R., Mottram JC., Navarro M., Rigden DJ., Romano PS., Stoka V., Turk B., Michels PAM. 2011. Autophagy in protists. Autophagy 7:1-32.

Eisen JA., Coyne RS., Wu M., Wu D., Thiagarajan M., Wortman JR., Badger JH., Ren Q., Amedeo P., Jones KM., Tallon LJ., Delcher AL., Salzberg SL., Silva JC., Haas BJ., Majoros WH., Farzad M., Carlton JM., Smith RK., Garg J., Pearlman RE., Karrer KM., Sun L., Manning G., Elde NC., Turkewitz AP., Asai DJ., Wilkes DE., Wang Y., Cai H., Collins K., Stewart BA., Lee SR., Wilamowska K., Weinberg Z., Ruzzo WL., Wloga D., Gaertig J., Frankel J., Tsao C-C., Gorovsky M a., Keeling PJ., Waller RF., Patron NJ., Cherry JM., Stover N a., Krieger CJ., del Toro C., Ryder HF., Williamson SC., Barbeau R a., Hamilton EP., Orias E. 2006. Macronuclear genome sequence of the ciliate Tetrahymena thermophila, a model eukaryote. PLoS Biology 4:e286. DOI: 10.1371/journal.pbio.0040286.

Feng Y., He D., Yao Z., Klionsky DJ. 2014. The machinery of macroautophagy. Cell Research 24:24-41. DOI: 10.1038/cr.2013.168.

Field HI., Coulson RMR., Field MC. 2013. An automated graphics tool for comparative genomics: the Coulson plot generator. BMC Bioinformatics 14:141. DOI: 10.1186/1471- 
536

537

538

539

540

541

542

543

544

545

546

547

548

549

550

551

552

553

554

555

556

557

558

559

560

561

562

563

564

565

566

567

568

569

570

571

572

573

574

2105-14-141.

Finn RD., Bateman A., Clements J., Coggill P., Eberhardt RY., Eddy SR., Heger A., Hetherington K., Holm L., Mistry J., Sonnhammer ELL., Tate J., Punta M. 2014. Pfam: The protein families database. Nucleic Acids Research 42:222-230. DOI: 10.1093/nar/gkt1223.

Finn RD., Clements J., Eddy SR. 2011. HMMER web server: Interactive sequence similarity searching. Nucleic Acids Research 39:29-37. DOI: 10.1093/nar/gkr367.

Földvári-Nagy L., Ari E., Csermely P., Korcsmáros T., Vellai T. 2014. Starvation-response may not involve Atg1-dependent autophagy induction in non-unikont parasites. Scientific Reports 4:5829. DOI: 10.1038/srep05829.

van Gurp M., Festjens N., van Loo G., Saelens X., Vandenabeele P. 2003. Mitochondrial intermembrane proteins in cell death. Biochemical and Biophysical Research Communications 304:487-497. DOI: 10.1016/S0006-291X(03)00621-1.

Jiang P., Mizushima N. 2014. Autophagy and human diseases. Cell Research 24:69-79.

Jiang Q., Zhao L., Dai J., Wu Q. 2012. Analysis of autophagy genes in microalgae: Chlorella as a potential model to study mechanism of autophagy. PLOS ONE 7:1-16. DOI: 10.1371/journal.pone.0041826.

Kabeya Y., Noda NN., Fujioka Y., Suzuki K., Inagaki F., Ohsumi Y. 2009. Characterization of the Atg17-Atg29-Atg31 complex specifically required for starvation-induced autophagy in Saccharomyces cerevisiae. Biochemical and Biophysical Research Communications 389:612-615. DOI: 10.1016/j.bbrc.2009.09.034.

Kang R., Zeh HJ., Lotze MT., Tang D. 2011. The Beclin 1 network regulates autophagy and apoptosis. Cell Death and Differentiation 18:571-580. DOI: 10.1038/cdd.2010.191.

Kihara A., Noda T., Ishihara N., Ohsumi Y. 2001. Two distinct Vps34 phosphatidylinositol 3kinase complexes function in autophagy and carboxypeptidase y sorting in Saccharomyces cerevisiae. Journal of Cell Biology 153:519-530. DOI: 10.1083/jcb.152.3.519.

Kobayashi T., Endoh H. 2005. A possible role of mitochondria in the apoptotic-like programmed nuclear death of Tetrahymena thermophila. The FEBS Journal 272:5378-87. DOI: 10.1111/j.1742-4658.2005.04936.x.

Letunic I., Bork P. 2016. Interactive Tree Of Life (iTOL): An online tool for phylogenetic tree display and annotation. Nucleic Acids Research 23:127-128. DOI: 10.1093/bioinformatics/btl529.

Li M., Hou Y., Wang J., Chen X., Shao ZM., Yin XM. 2011. Kinetics comparisons of mammalian Atg4 homologues indicate selective preferences toward diverse Atg8 substrates. Journal of Biological Chemistry 286:7327-7338. DOI: 10.1074/jbc.M110.199059.

Liang C., Feng P., Ku B., Dotan I., Canaani D., Oh B-H., Jung JU. 2006. Autophagic and tumour suppressor activity of a novel Beclin1-binding protein UVRAG. Nature Cell Biology 8:688699. DOI: $10.1038 /$ ncb1426.

Liu ML., Yao MC. 2012. Role of ATG8 and Autophagy in programmed nuclear degradation in Tetrahymena thermophila. Eukaryotic Cell 11:494-506. DOI: 10.1128/EC.05296-11. 
575 Lynch-Day M a., Klionsky DJ. 2010. The Cvt pathway as a model for selective autophagy. FEBS Letters 584:1359-1366. DOI: 10.1016/j.febslet.2010.02.013.

577

578

579

580

581

582

583

584

585

586

587

588

589

590

591

592

593

594

595

596

597

598

599

600

601

602

603

604

605

606

607

608

609

610

611

612

613

614

MacColl E., Therkelsen MD., Sherpa T., Ellerbrock H., Johnston L a., Jariwala RH., Chang W., Gurtowski J., Schatz MC., Mozammal Hossain M., Cassidy-Hanley DM., Clark TG., Chang W-J. 2015. Molecular genetic diversity and characterization of conjugation genes in the fish parasite Ichthyophthirius multifiliis. Molecular Phylogenetics and Evolution 86:1-7. DOI: 10.1016/j.ympev.2015.02.017.

Marchler-Bauer a., Derbyshire MK., Gonzales NR., Lu S., Chitsaz F., Geer LY., Geer RC., He J., Gwadz M., Hurwitz DI., Lanczycki CJ., Lu F., Marchler GH., Song JS., Thanki N., Wang Z., Yamashita R a., Zhang D., Zheng C., Bryant SH. 2014. CDD: NCBI's conserved domain database. Nucleic Acids Research 43:D222-D226. DOI: 10.1093/nar/gku1221.

Marino G., Uria JA., Puente XS., Quesada V., Bordallo J., Lopez-Otin C. 2003. Human autophagins, a family of cysteine proteinases potentially implicated in cell degradation by autophagy. Journal of Biological Chemistry 278:3671-3678. DOI: 10.1074/jbc.M208247200.

Mccarthy DJ., Smyth GK. 2009. Testing significance relative to a fold-change threshold is a TREAT. Bioinformatics 25:765-771. DOI: 10.1093/bioinformatics/btp053.

Miao W., Xiong J., Bowen J., Wang W., Liu Y., Braguinets O., Grigull J., Pearlman RE., Orias E., Gorovsky M a. 2009. Microarray analyses of gene expression during the Tetrahymena thermophila life cycle. Plos ONE 4:e4429. DOI: 10.1371/journal.pone.0004429.

Mizushima N., Kuma A., Kobayashi Y., Yamamoto A., Matsubae M., Takao T., Natsume T., Ohsumi Y., Yoshimori T. 2003. Mouse Apg16L, a novel WD-repeat protein, targets to the autophagic isolation membrane with the Apg12-Apg5 conjugate. Journal of Cell Science 116:1679-1688. DOI: $10.1242 /$ jcs.00381.

Mizushima N., Yoshimori T. 2007. How to Interpret LC3 Immunoblotting. Autophagy 3:4-7. DOI: $10.4161 /$ auto.4600.

Mizushima N., Yoshimori T., Ohsumi Y. 2011. The Role of Atg Proteins in Autophagosome Formation. Annual Review of Cell and Developmental Biology 27:107-132. DOI: 10.1146/annurev-cellbio-092910-154005.

Molejon MI., Ropolo A., Re A Lo., Boggio V., Vaccaro MI. 2013. The VMP1-Beclin 1 interaction regulates autophagy induction. Scientific Reports 3:1055. DOI: 10.1038/srep01055.

Nilsson JR. 1984. On starvation-induced autophagy in Tetrahymena. Carlsberg Research Communications. 49:323-340.

Obara K., Sekito Y., Ohsumi Y. 2006. Assortment of Phosphatidylinositol 3-Kinase Complexes-Atg14p Directs Association of Complex I to the Pre-autophagosomal Structure in Saccharomyces cerevisiae. Molecular Biology of the Cell 17:1018-1032. DOI: 10.1091/mbc.E05.

Orias E., Cervantes MD., Hamilton EP. 2011. Tetrahymena thermophila, a unicellular eukaryote with separate germline and somatic genomes. Research in Microbiology 162:578-86. DOI: 
615

616

617

618

619

620

621

622

623

624

625

626

627

628

629

630

631

632

633

634

635

636

637

638

639

640

641

642

643

644

645

646

647

648

649

650

$$
\text { 10.1016/j.resmic.2011.05.001. }
$$

Osada E., Akematsu T., Asano T., Endoh H. 2014. A novel mitochondrial nuclease-associated protein: A major executor of the programmed nuclear death in Tetrahymena thermophila. Biology of the Cell 106:97-109. DOI: 10.1111/boc.201300037.

Rigden DJ., Michels P a., Ginger ML. 2009. Autophagy in protists: Examples of secondary loss, lineage-specific innovations, and the conundrum of remodeling a single mitochondrion. Autophagy 5:784-794. DOI: 8838 [pii].

Ropolo A., Grasso D., Pardo R., Sacchetti ML., Archange C., Lo Re A., Seux M., Nowak J., Gonzalez CD., Iovanna JL., Vaccaro MI. 2007. The pancreatitis-induced vacuole membrane protein 1 triggers autophagy in mammalian cells. Journal of Biological Chemistry 282:37124-37133. DOI: 10.1074/jbc.M706956200.

Shibutani ST., Yoshimori T. 2014. A current perspective of autophagosome biogenesis. Cell Research 24:58-68. DOI: 10.1038/cr.2013.159.

Slobodkin MR., Elazar Z. 2013. The Atg8 family: multifunctional ubiquitin-like key regulators of autophagy. Essays in Biochemistry. 55:51-64. DOI: 10.1042/bse0550051.

Swart EC., Bracht JR., Magrini V., Minx P., Chen X., Zhou Y., Khurana JS., Goldman AD., Nowacki M., Schotanus K., Jung S., Fulton RS., Ly A., McGrath S., Haub K., Wiggins JL., Storton D., Matese JC., Parsons L., Chang WJ., Bowen MS., Stover N a., Jones T a., Eddy SR., Herrick G a., Doak TG., Wilson RK., Mardis ER., Landweber LF. 2013. The Oxytricha trifallax Macronuclear Genome: A Complex Eukaryotic Genome with 16,000 Tiny Chromosomes. PLoS Biology 11. DOI: 10.1371/journal.pbio.1001473.

Williams R a M., Woods KL., Juliano L., Mottram JC., Coombs GH. 2009. Characterization of unusual families of ATG8-like proteins and ATG12 in the protozoan parasite Leishmania major. Autophagy 5:159-172. DOI: 10.4161/auto.5.2.7328.

Woehrer SL., Aronica L., Suhren JH., Busch CJ-L., Noto T., Mochizuki K. 2015. A Tetrahymena Hsp90 co-chaperone promotes siRNA loading by ATP-dependent and ATPindependent mechanisms. The EMBO Journal 34:559-77. DOI: 10.15252/embj.201490062.

Xie Z., Klionsky DJ. 2007. Autophagosome formation: core machinery and adaptations. Nature Cell Biology 9:1102-1109. DOI: 10.1038/ncb1007-1102.

Yamaguchi M., Noda NN., Yamamoto H., Shima T., Kumeta H., Kobashigawa Y., Akada R., Ohsumi Y., Inagaki F. 2012. Structural Insights into Atg10-Mediated Formation of the Autophagy-Essential Atg12-Atg5 Conjugate. Structure 20:1244-1254. DOI: 10.1016/j.str.2012.04.018.

Figures and legends 


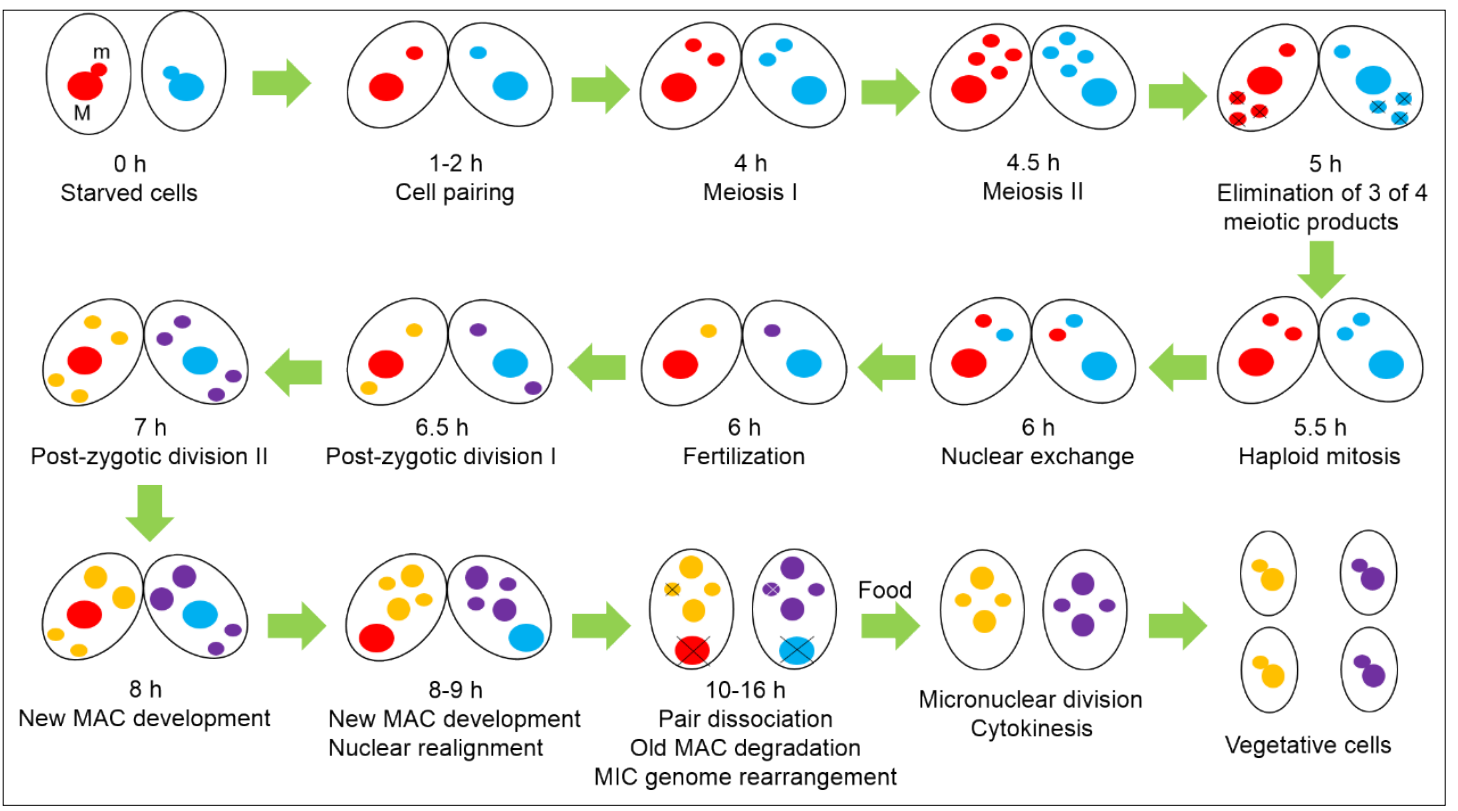

652 Figure 1- Life cycle and nuclear events during conjugation in Tetrahymena thermophila.

653 In the absence of sufficient nutrients, two cells of different mating types begin conjugation; the

654 sexual reproduction stage of Tetrahymena. During conjugation, a number of nuclear events take 655 place, including meiosis of the micronucleus $(\mathrm{m})$ to yield four meiotic products, elimination of 656 three of four of these products, haploid mitosis of the remaining meiotic product, nuclear exchange 657 and fertilization, post-zygotic divisions of the fertilized nucleus, new macronucleus (M) 658 development and elimination of the parental macronucleus in an apoptotic and autophagic fashion.

659 When nutrients are available, exconjugants undergo cytokinesis and turn into vegetative cells. 660 Approximate timing indicates time after mixing (hours) different mating types of starved cells 661 (Miao et al., 2009). 


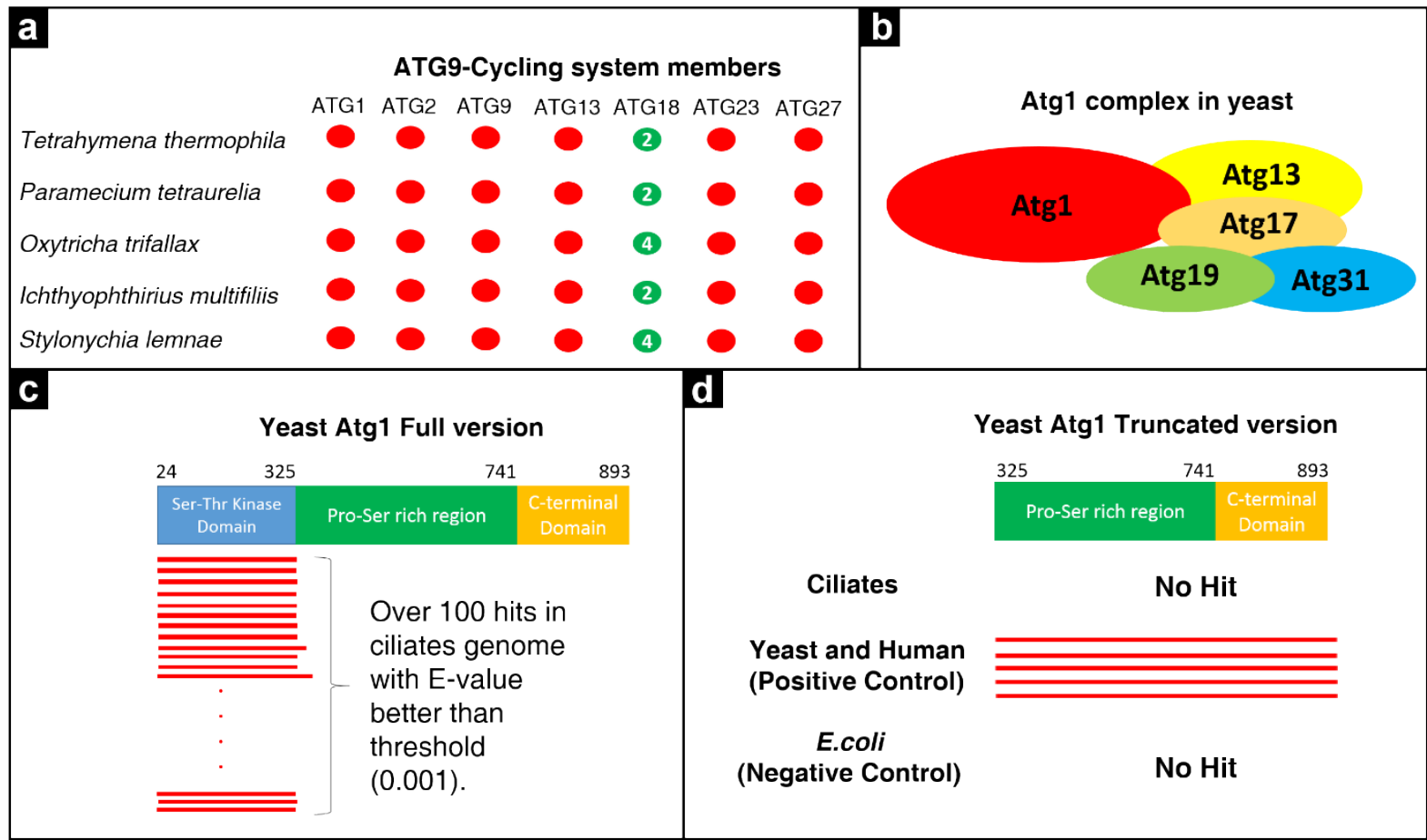

664 Figure 2- Analysis of Atg9-Cycling system members in ciliates.

665 a) Distribution of system members among ciliates. Red circles denote the absence of indicated

666 genes in the indicated species. Green circles show the presence of the indicated genes in the

667 indicated species. A digit in the green circles refers to the number of significant hits.

668 b) Schematic depiction of Atg1 complex in yeast. Atg1 interacts with other components of the

669 complex via its C-terminal domain. A cartoon was constructed based on information described in

670 Földvári-Nagy L et al., 2014.

671 c) PSI-BLAST analysis of Atg1 in ciliate genomes using yeast Atg1. Significant hits only cover

672 the N-terminal domain of the Atg1.

673 d) PSI-BLAST analysis of Atg1 in ciliate genomes using truncated version of yeast Atg1 which

674 does not include the N-terminal domain. No significant hits were obtained in ciliates. Yeast and

675 human genomes were also probed with the same truncated sequence and significant hits were 
676 observed (positive control). No hits were retrieved from the E.coli K12 genome as expected

677 (negative control).

678

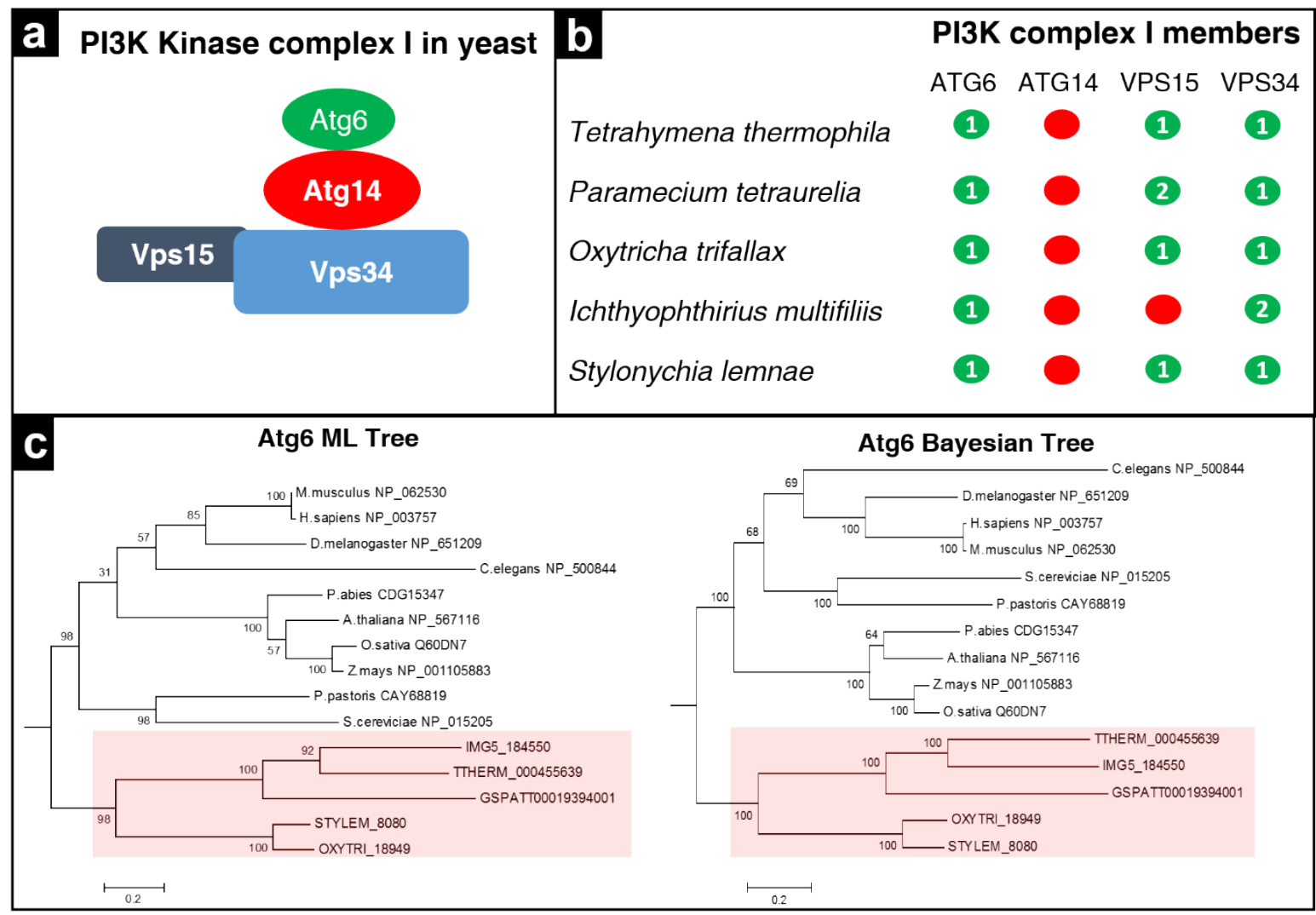

680 Figure 3- Analysis of PI3K complex members in ciliates.

681 a) Schematic model of PI3K Kinase complex in yeast according to Ref. 20.

682 b) Distribution of complex members among ciliates as in Fig 2.

683 c) Maximum likelihood (ML) tree and Bayesian tree of Atg6. Trees were computed based on

684 multiple alignment of Atg6 catalytic domains. Both trees show the same placement of critical

685 nodes. Ciliate Atg6s were highlighted with pink color. 


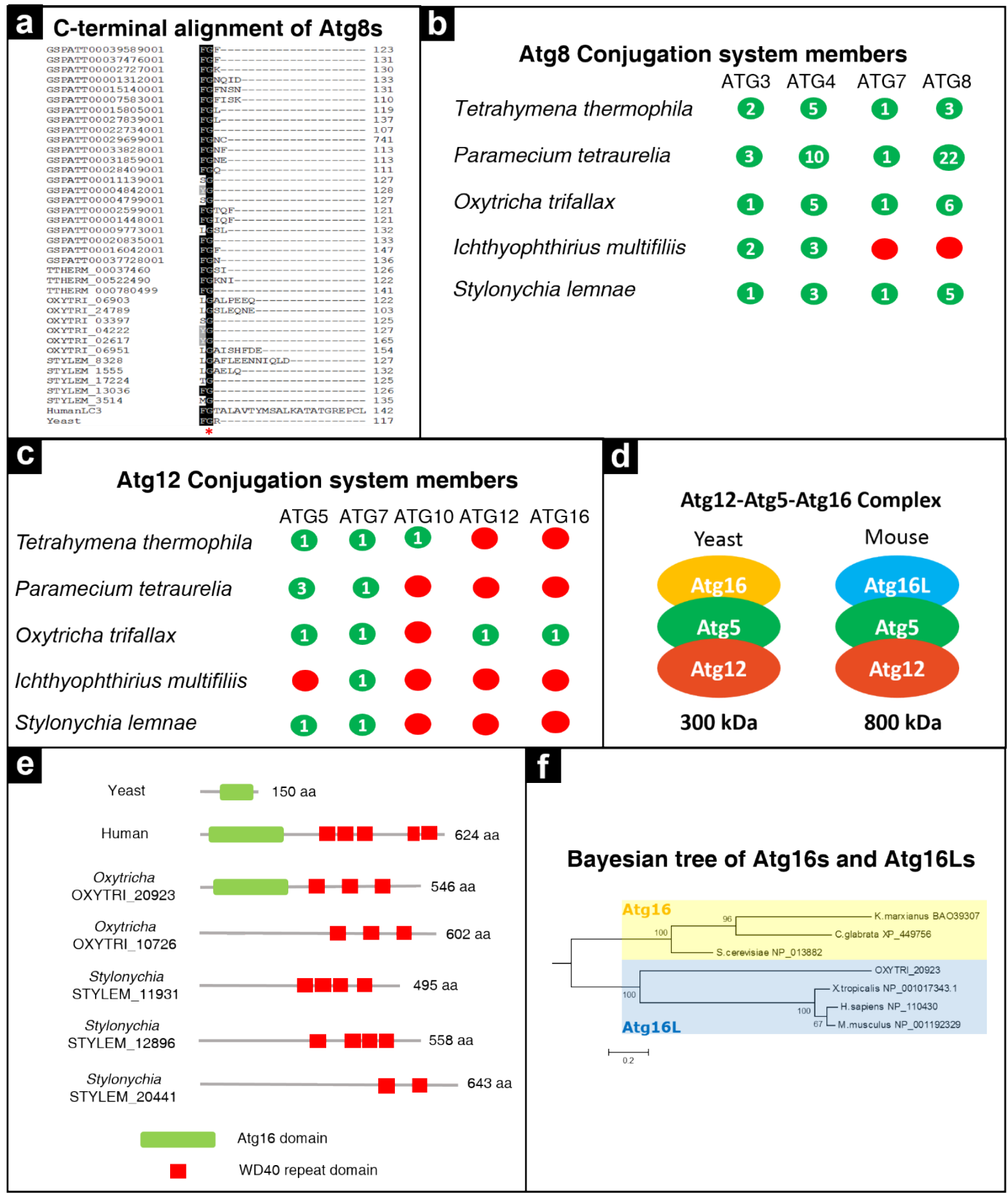

686

ATG5 ATG7 ATG10 ATG12 ATG16

Atg12-Atg5-Atg16 Complex

(1)

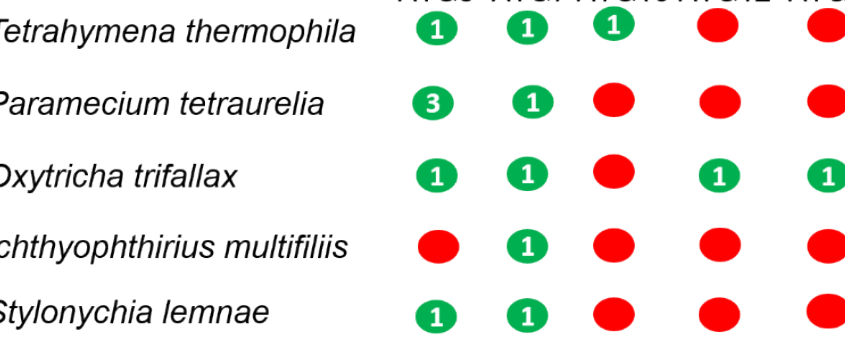

687 Figure 4- Analysis of ubiquitin-like conjugation system members in ciliates. 
688 a) Multiple sequence alignment of C-terminal region of Atg8s. A red asterisk indicates the 689 conserved glycine residue. Distribution of Atg8 (b) and Atg12 (c) conjugation system members 690 among ciliates, as in Fig 2.

691 d) Schematic depiction of Atg12-Atg5-Atg16 complex in yeast and mouse.

692 e) Domain analysis of Atg16 and Atg16L candidates in Oxytricha and Stylonychia. Accession 693 numbers; yeast Atg16 (NP_013882) and human Atg16L (XP_011547155).

694 f) Bayesian tree of Atg16s and Atg16Ls. Tree was computed based on multiple alignment of Atg16 695 domains of yeast Atg16s and mammalian and reptile Atg16s. Tree shows that OXYTRI_20923 696 from Oxytricha is an Atg16L ortholog not Atg16.

697

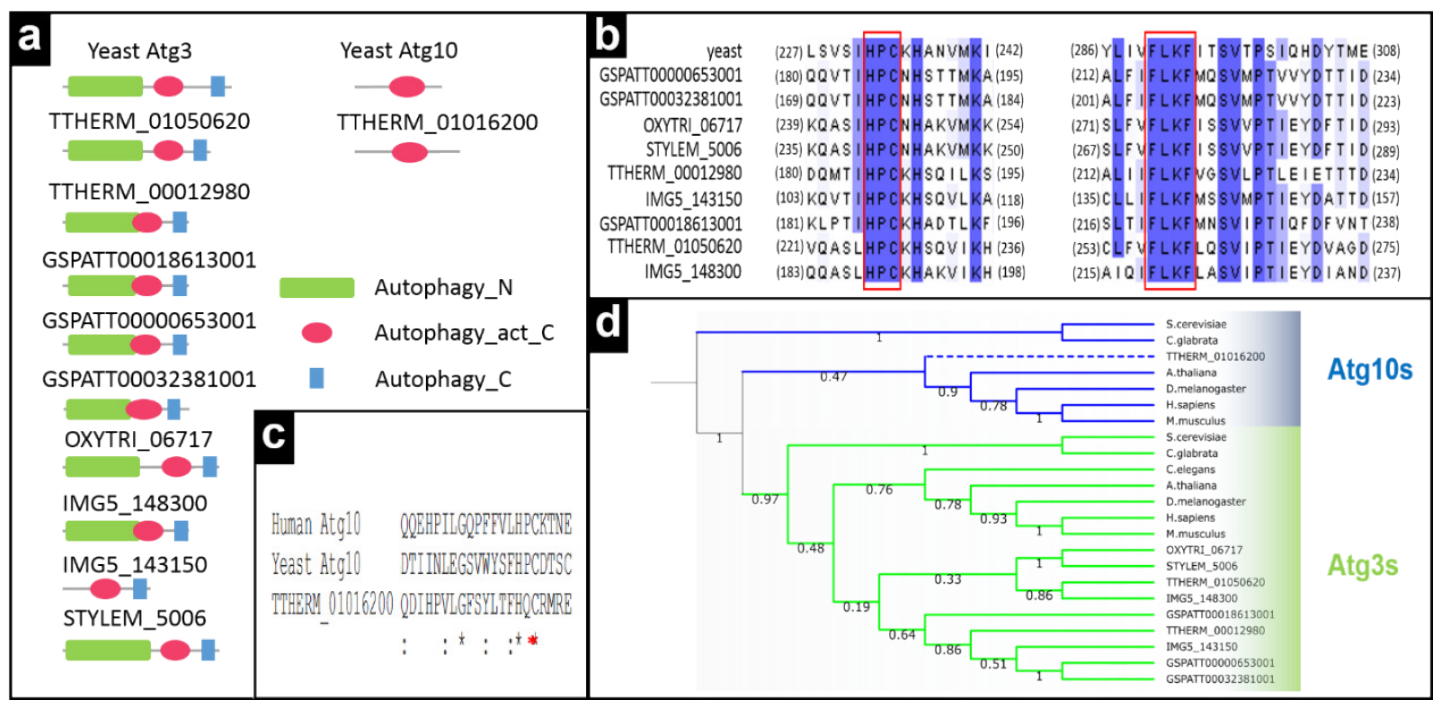

699 Figure 5 - In silico characterization of Atg3 and Atg10 in ciliates.

700 a) Domain analysis of Atg3 and Atg10.

701 b) Partial multiple alignment of Atg3s in ciliates. Atg3-specific conserved motifs were boxed with 702 red rectangles.

703 c) Partial multiple alignment of the region containing catalytic cysteine (Red asterisk) residues of

704 Tetrahymena Atg10-like protein with yeast and human Atg10 orthologues. 
a
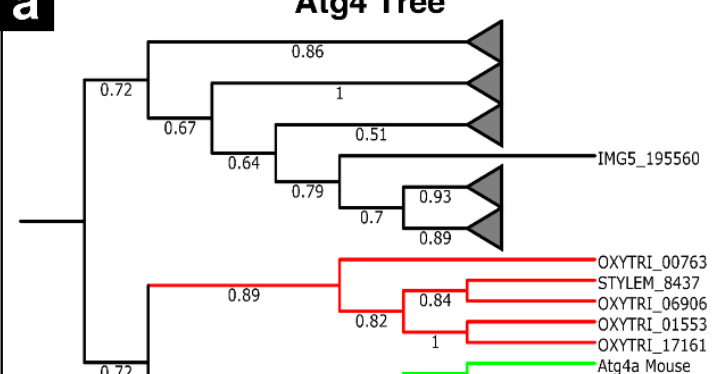

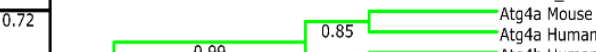

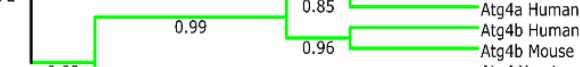

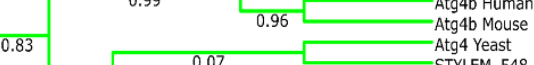

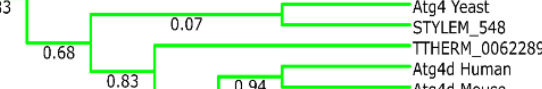

$0.94 \square$ Atg $4 \mathrm{~d}$ Human

$0.99 \square$ Atg 4c Mouse
Atg4 Tree

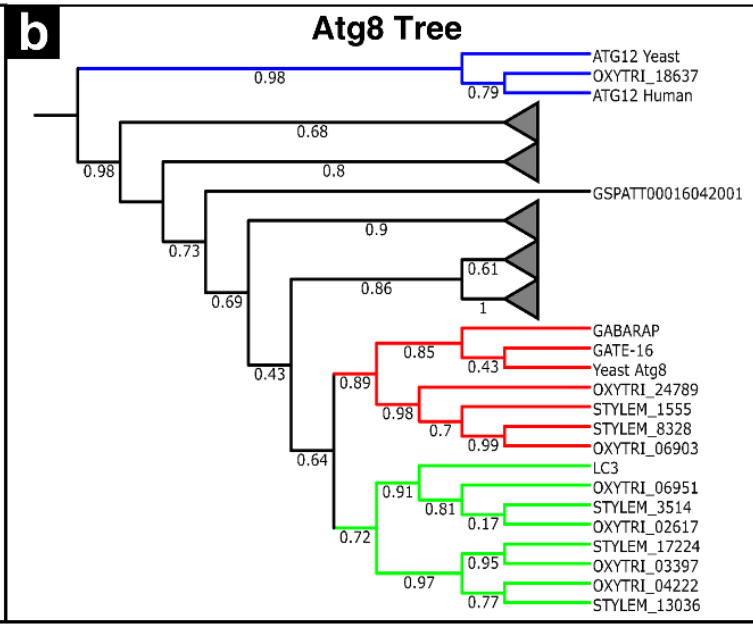

709

Figure 6- Phylogenetic analysis of ciliate Atg4s, and Atg8s.

710 Maximum likelihood trees were calculated for Atg4s (a) and Atg8s (b) as described in the

711 Materials \& Methods section. Bootstrap values are given at each node and branch lengths in the

712 tree were ignored. Ciliate-specific clades were collapsed and shown as triangles.

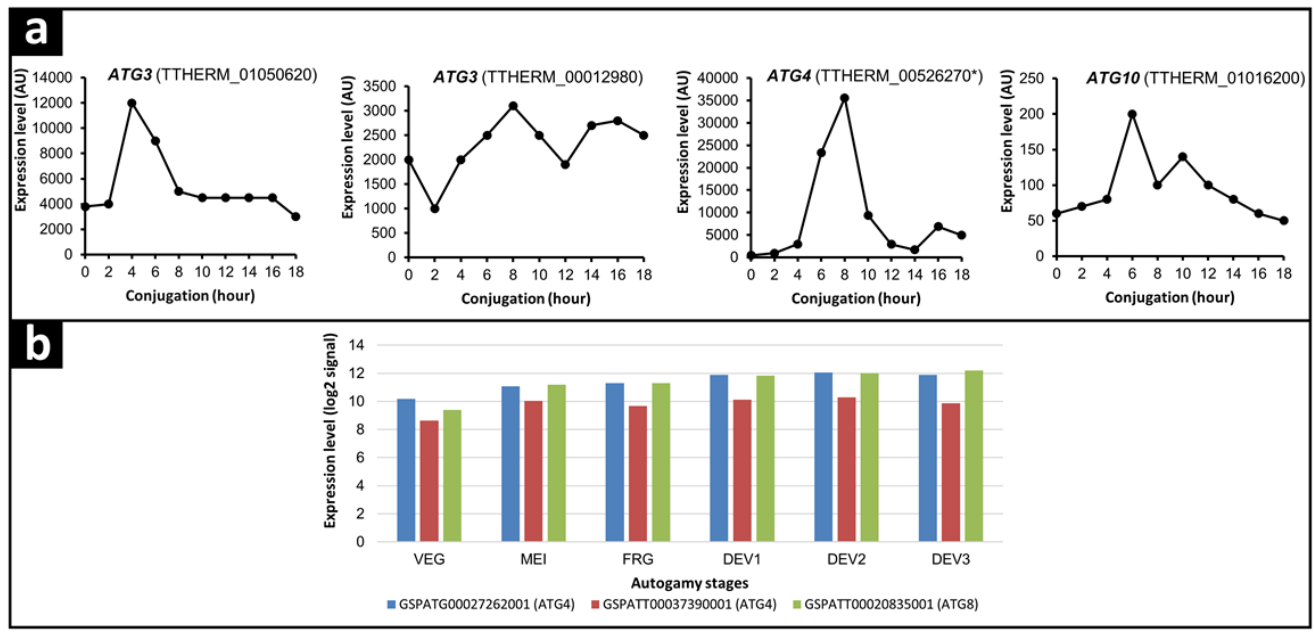


715 Figure 7- mRNA expression analysis of ATG genes upregulated during Tetrahymena

716 conjugation and Paramecium autogamy.

717 a) Data shows the mRNA expression profile of $A T G 3 \mathrm{~s}, A T G 4$ and $A T G 10$ during conjugation of

718 Tetrahymena. The profiles are obtained from the Tetrahymena Functional Genomics Database-

719 TetraFGD (http://tfgd.ihb.ac.cn/). Data represents the average of the results obtained by Gorowski,

720 Miao and Pearlman Labs (Miao et al., 2009). *Note that TTHERM_00526270 contains two genes;

721 one COI3 (transcript id: gene_000015720), and the other, Atg4 (C54 peptidase domain, transcript

722 id: gene_000006383) (Woehrer et al., 2015). We focused on the latter transcript in this study.

723 b) Data shows the mRNA expression profile of $A T G 4 s$ and $A T G 8$ during autogamy of

724 Paramecium. The profiles are obtained from the Paramecium Database (Paramecium DB) at

725 http://paramecium.cgm.cnrs-gif.fr/. The time course of autogamy was characterized by nuclear

726 morphology as follows: VEG; log-phase vegetative cells showing no signs of meiosis. MEI;

727 beginning of macronuclear fragmentation and micronuclear meiosis. FRG; population in which

728 about $50 \%$ of cells have a fragmented old macronucleus.DEV1; earliest stage at which a significant

729 proportion of cells has visible macronuclear anlage.DEV2; majority of cells with macronuclear

730 anlage.DEV3; population of cells ten hours after DEV2. Expression levels are shown as log2

731 values of raw measurements (Arnaiz et al., 2010).

732 


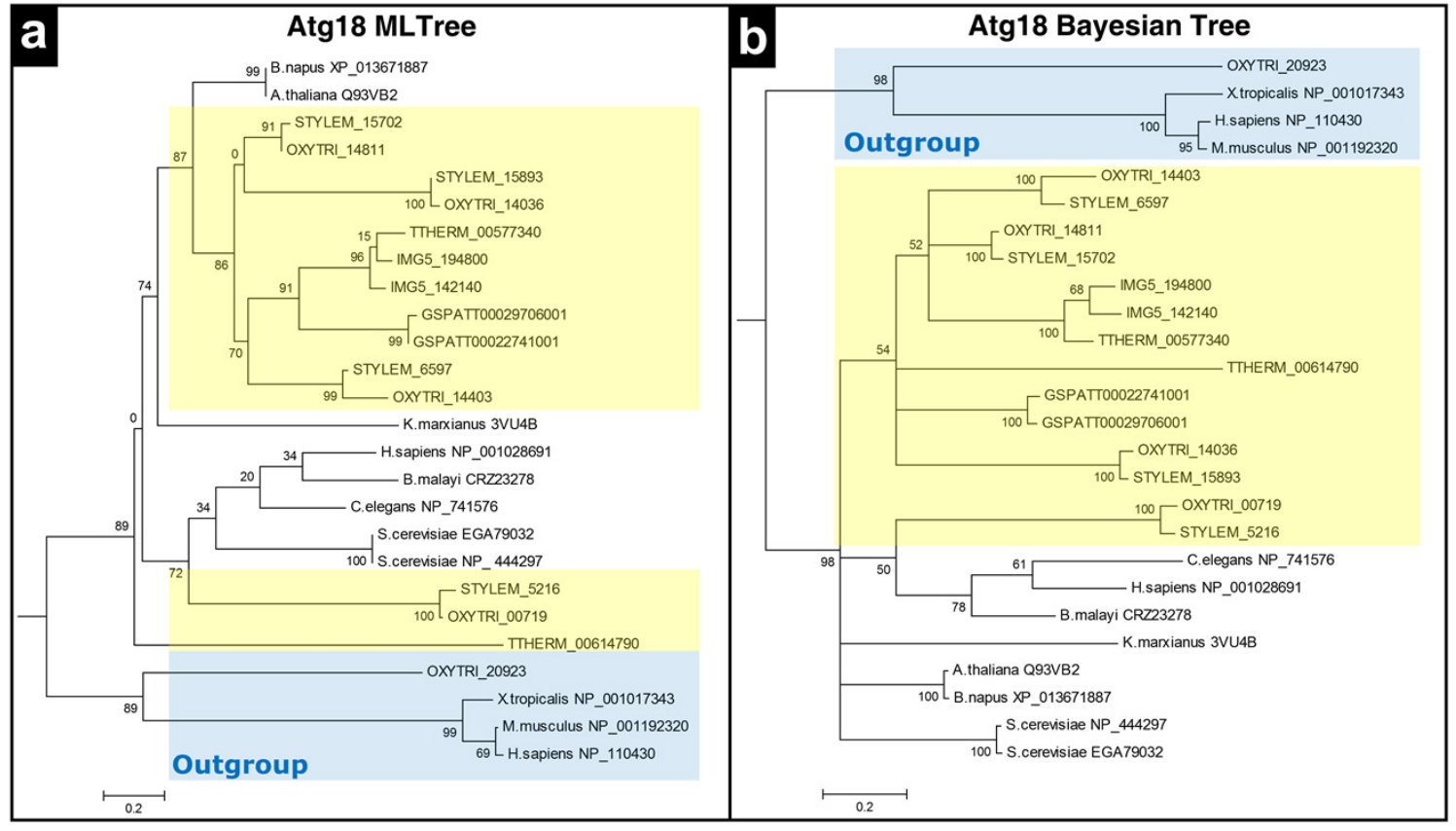

734 Fig. S1: Phylogenetic analyses of Atg18s in ciliates. a) Maximum likelihood (ML) tree. b)

735 Bayesian tree. Trees were computed based on multiple alignment of WD40 domains of Atg18 and

736 Atg16L proteins. Since Atg16L proteins contain WD40 repeats like Atg18 proteins, trees were

737 rooted with WD40 domain of a set of Atg16L proteins including one from Oxytricha

738 (OXYTRI_209239) (blue box). Ciliate members were highlighted with light orange color. Both

739 trees show the same placement of critical nodes. 


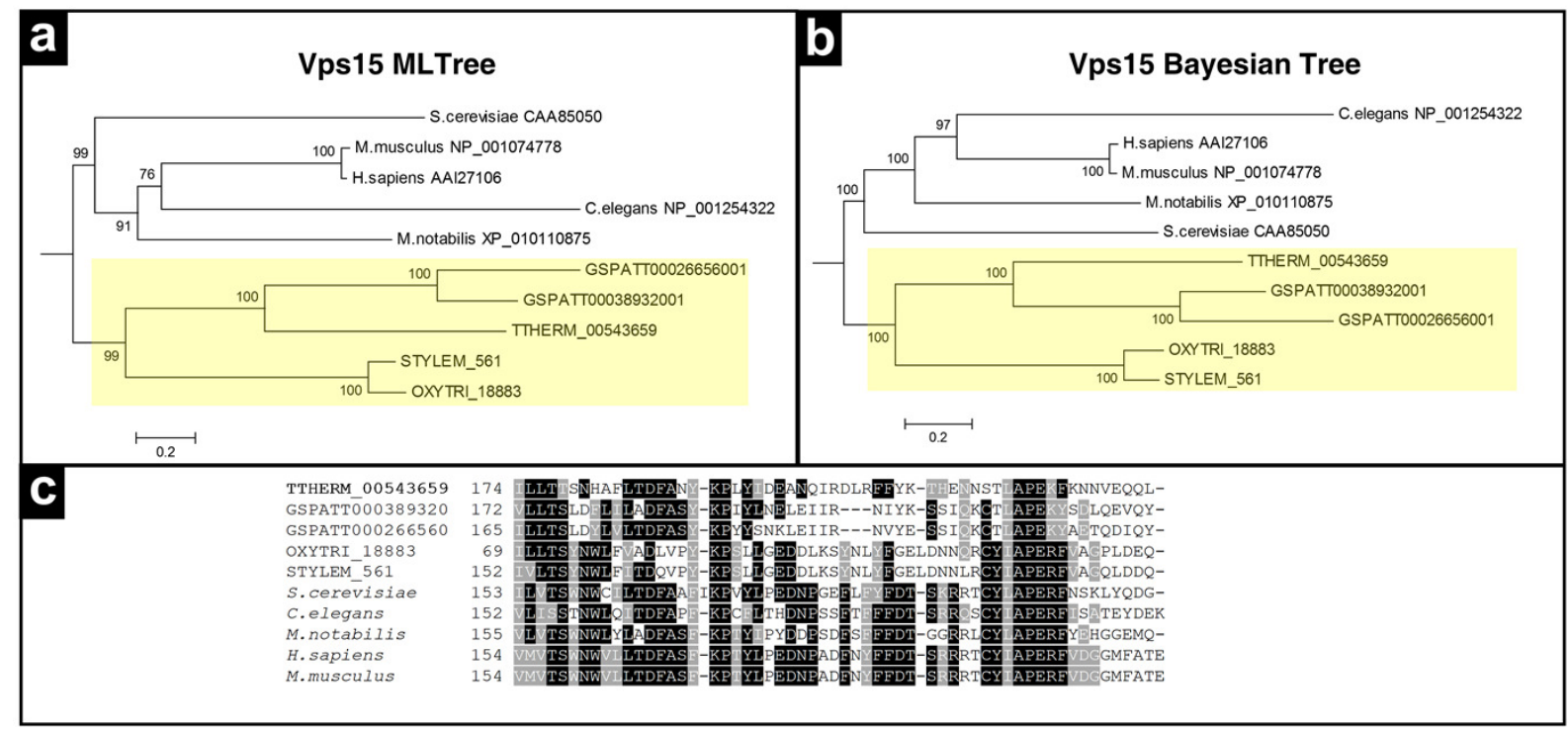

741 Fig. S2: Phylogenetic analyses of Vps15s in ciliates. a) Maximum likelihood (ML) tree. b)

742 Bayesian tree. Ciliate members were highlighted. Both trees show the same placement of critical nodes. c) Partial multiple alignment of Vps15 proteins from ciliates and various model organisms.

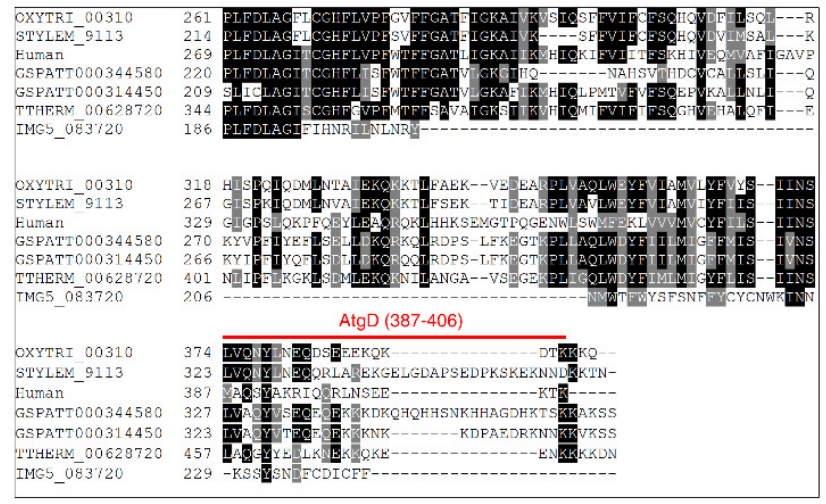

Figure S3: Partial multiple alignment of ciliate Vmp1 proteins. It has been shown that human

746 Vmp1 protein (CAG38552.1) interacts with Beclin-1 through its C-terminal located autophagy

747 (AtgD) domain to regulate autophagy induction (Molejon et al., 2013). AtgD domain of Human

748 Vmp1 (387-406) is marked with red line. Last 20 amino acids of Human Vmp1 is partially 749 conserved in ciliates. 


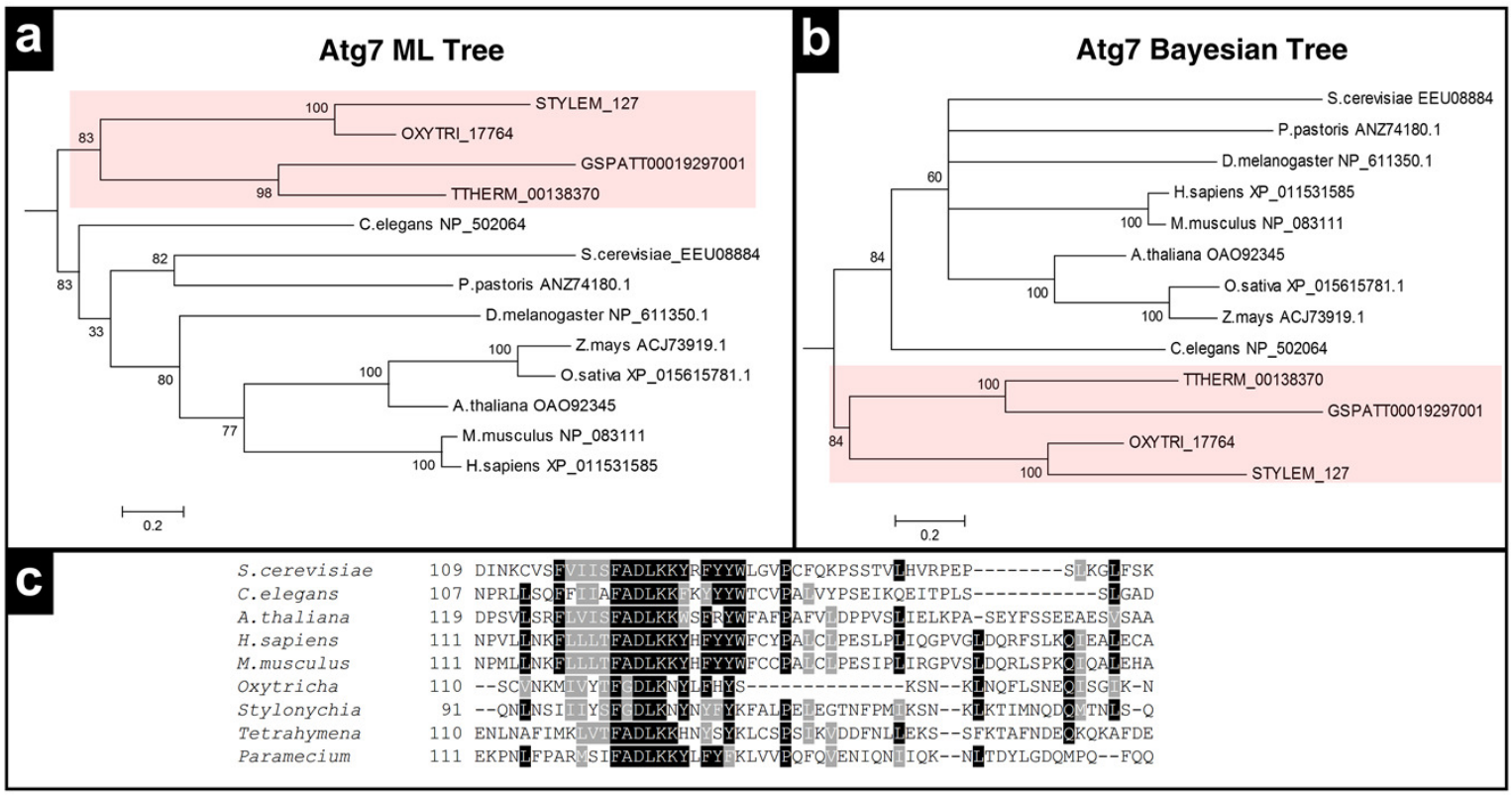

751 Fig. S4: Phylogenetic analyses of Atg7s in ciliates. a) Maximum likelihood (ML) tree. b)

752 Neighbor joining (NJ) tree. Trees were computed based on multiple alignment of N-terminal Atg7

753 domains. Both trees show the same placement of critical nodes. Ciliate members were highlighted

754 with pink color c) Partial multiple alignment of Atg7 proteins from ciliates and various model organisms. 


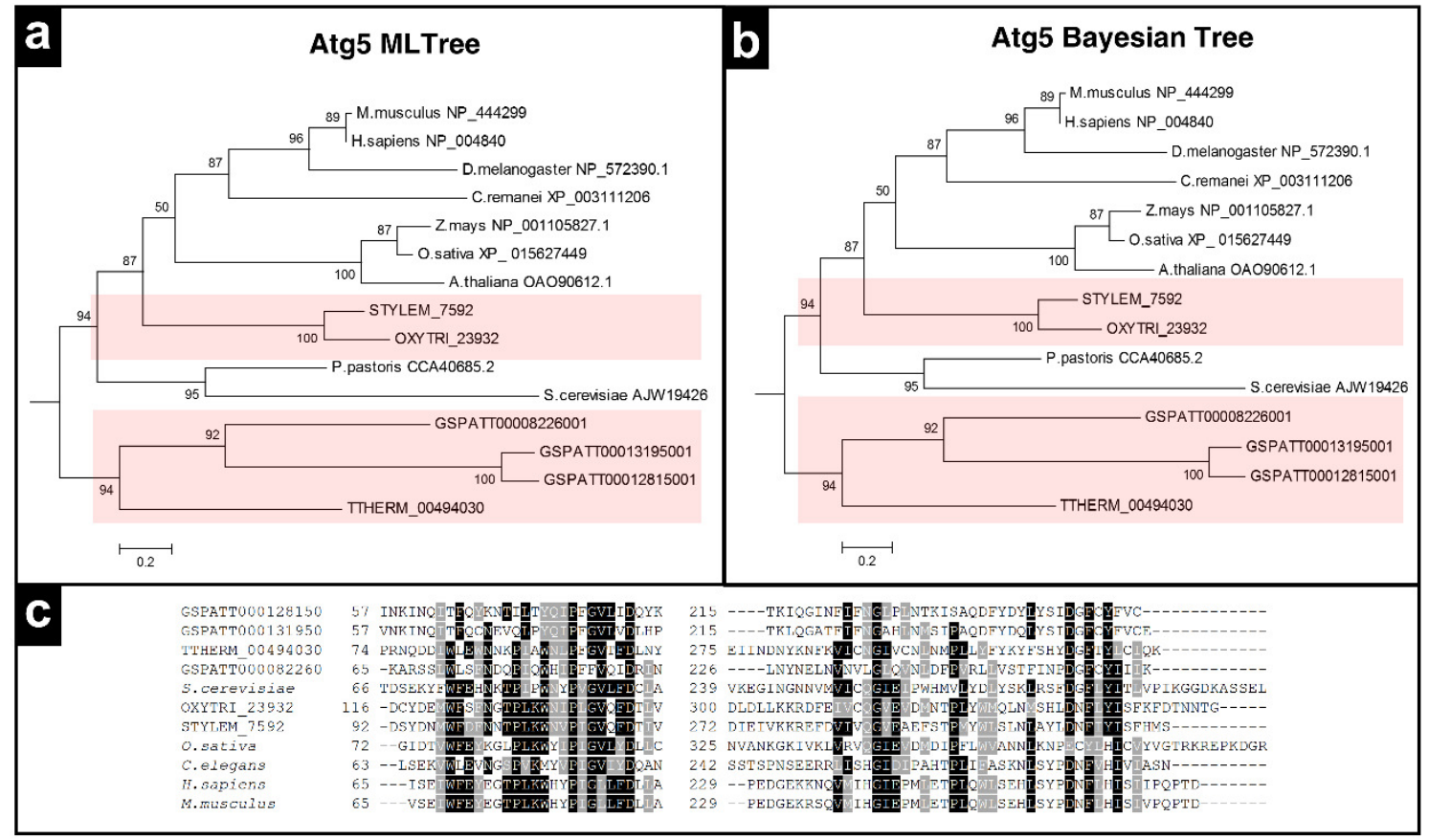

757 Fig. S5: Phylogenetic analyses of Atg5s in ciliates. a) Maximum likelihood (ML) tree. b)

758 Bayesian tree. Trees were computed based on multiple alignment of Atg5 domains. Ciliate 759 members were highlighted with pink color. Both trees show the same placement of critical nodes. c) Partial multiple alignment of Atg5 proteins from ciliates and various model organisms.
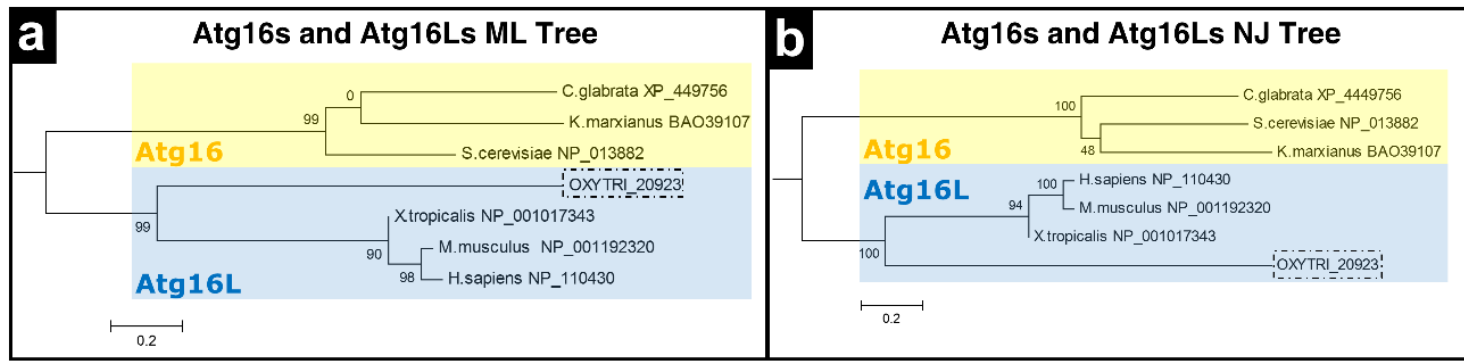

762 Fig. S6: OXYTRI_20923 from Oxytricha is an Atg16L but not Atg16 protein. Phylogenetic

763 analyses of Atg16s and Atg16Ls. a) Maximum likelihood (ML) tree. b) Neighbor joining tree.

764 Trees were computed based on multiple alignment of Atg16 domains of yeast Atg16s and 
765 mammalian and reptile Atg16s. Both trees show that OXYTRI_20923 from Oxytricha (dotted box)

766 is an Atg16L ortholog not Atg16.

767

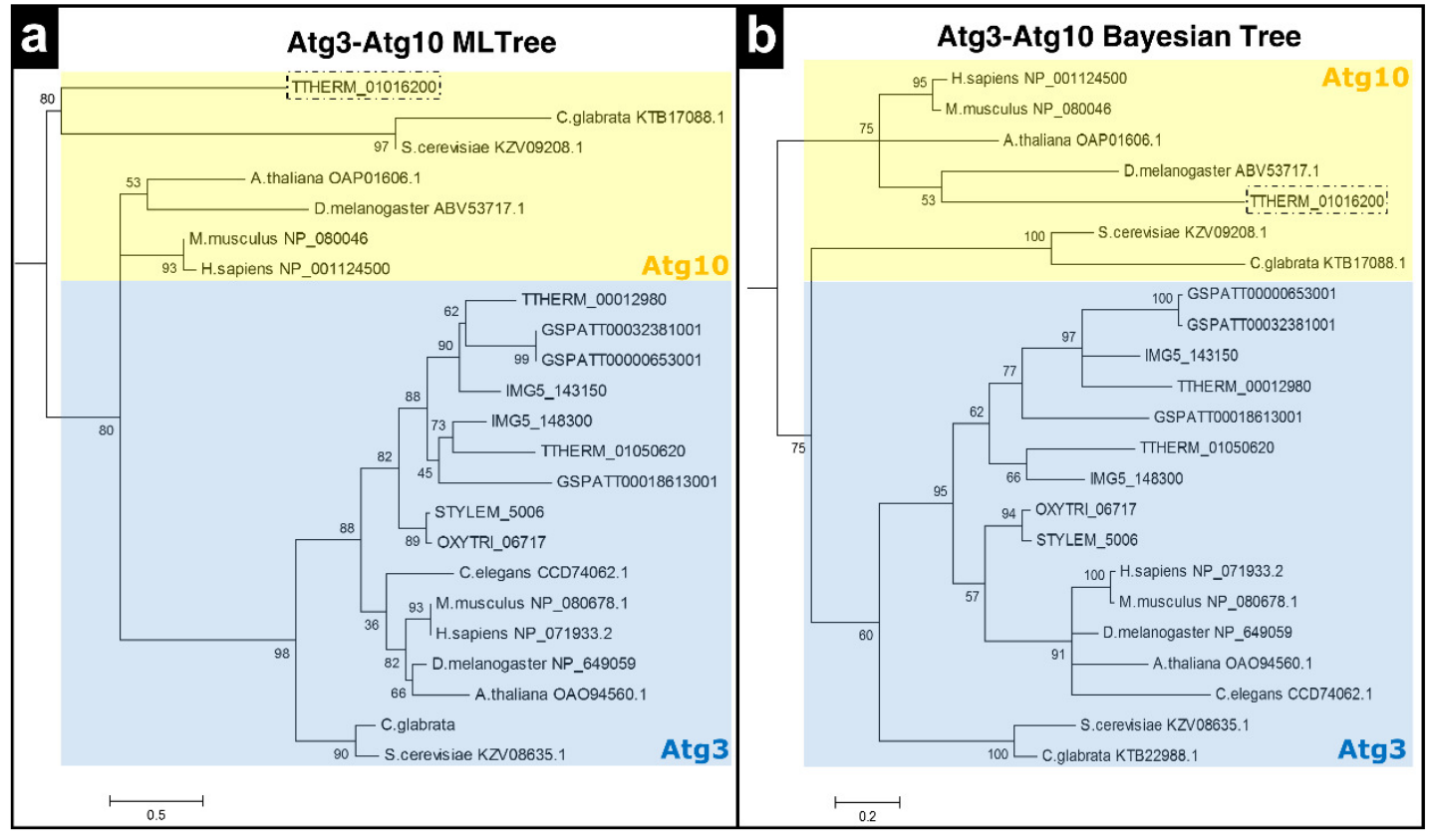

768 Fig. S7: Tetrahymena TTHERM_00012980 is the sole Atg10 ortholog in ciliates. Phylogenetic

769 analyses of Atg3s and Atg10s. a) Maximum likelihood (ML) tree. b) Bayesian tree. Trees were

770 computed based on multiple alignment of Autophagy_act_C domains (PF03987) of Atg3s and

771 Atg10s. Both trees show that TTHERM_00012980 from Tetrahymena (dotted box) is the sole

772 Atg10 ortholog in ciliates. 